\title{
Pseudomonas aeruginosa utilizes host polyunsaturated phosphatidylethanolamines to trigger theft-ferroptosis in bronchial epithelium
}

\author{
Haider H. Dar, ${ }^{1}$ Yulia Y. Tyurina, ${ }^{1}$ Karolina Mikulska-Ruminska, ${ }^{2,3}$ Indira Shrivastava, ${ }^{1,2}$ Hsiu-Chi Ting, ${ }^{1}$ Vladimir A. Tyurin, ${ }^{1}$ \\ James Krieger, ${ }^{2}$ Claudette M. St. Croix, ${ }^{4}$ Simon Watkins, ${ }^{4}$ Erkan Bayir, ${ }^{1}$ Gaowei Mao ${ }^{1,5}$ Catherine R. Armbruster, ${ }^{6}$ Alexandr Kapralov, ${ }^{1}$ \\ Hong Wang, ${ }^{7}$ Matthew R. Parsek, ${ }^{8}$ Tamil S. Anthonymuthu, ${ }^{1,5}$ Abiola F. Ogunsola, ${ }^{6}$ Becca A. Flitter, ${ }^{6}$ Cody J. Freedman, ${ }^{9}$ \\ Jordan R. Gaston, ${ }^{6}$ Theodore R. Holman, ${ }^{9}$ Joseph M. Pilewski, ${ }^{10}$ Joel S. Greenberger, ${ }^{11}$ Rama K. Mallampalli, ${ }^{10,12}$ Yohei Doi, ${ }^{10}$ \\ Janet S. Lee, ${ }^{10}$ Ivet Bahar, ${ }^{2}$ Jennifer M. Bomberger, ${ }^{6}$ Hülya Bayır,${ }^{1,5}$ and Valerian E. Kagan ${ }^{1,11,13,14,15}$
}

'Department of Environmental and Occupational Health and Center for Free Radical and Antioxidant Health and ${ }^{2}$ Department of Computational and System Biology, University of Pittsburgh, Pittsburgh, Pennsylvania, USA. Institute of Physics, Nicolaus Copernicus University, Torun, Poland. ${ }^{4}$ Department of Cell Biology, ${ }^{5}$ Department of Critical Care Medicine, ${ }^{6}$ Department of Microbiology and Molecular Genetics, and 'Department of Biostatistics, University of Pittsburgh, Pittsburgh, Pennsylvania, USA. ${ }^{8}$ Department of Microbiology, School of Medicine, University of Washington, Seattle, Washington, USA. ${ }^{9}$ Department of Chemistry and Biochemistry, University of California Santa Cruz, Santa Cruz, California, USA. ${ }^{10}$ Department of Medicine and "Department of Radiation Oncology, University of Pittsburgh, Pittsburgh, Pennsylvania, USA. ${ }^{2}$ Medical Specialty Service Line, Veterans Affairs Pittsburgh Healthcare System, Pittsburgh, Pennsylvania, USA. ${ }^{13}$ Department of Chemistry and ${ }^{14}$ Department of Pharmacology and Chemical Biology, University of Pittsburgh, Pittsburgh, Pennsylvania, USA. ${ }^{15}$ Laboratory of Navigational Redox Lipidomics, Institute of Regenerative Medicine, IM Sechenov Moscow State Medical University, Moscow, Russia.

Ferroptosis is a death program executed via selective oxidation of arachidonic acid-phosphatidylethanolamines (AA-PE) by 15-lipoxygenases. In mammalian cells and tissues, ferroptosis has been pathogenically associated with brain, kidney, and liver injury/diseases. We discovered that a prokaryotic bacterium, Pseudomonas aeruginosa, that does not contain AA-PE can express lipoxygenase ( $p$ LoxA), oxidize host AA-PE to 15-hydroperoxy-AA-PE (15-HOO-AA-PE), and trigger ferroptosis in human bronchial epithelial cells. Induction of ferroptosis by clinical $P$. aeruginosa isolates from patients with persistent lower respiratory tract infections was dependent on the level and enzymatic activity of pLoxA. Redox phospholipidomics revealed elevated levels of oxidized AA-PE in airway tissues from patients with cystic fibrosis (CF) but not with emphysema or CF without $P$. aeruginosa. We believe that the evolutionarily conserved mechanism of pLoxAdriven ferroptosis may represent a potential therapeutic target against $P$. aeruginosa-associated diseases such as CF and persistent lower respiratory tract infections.

\section{Introduction}

Ferroptosis is a cell death program that is executed via activation of a selective enzymatic process of lipid peroxidation (LPO) catalyzed by 15-lipoxygenases (15LOXes), dioxygenases for polyunsaturated fatty acids (PUFAs), under conditions where hydroperoxy-phospholipids (hydroperoxy-PLs) cannot be enzymatically inactivated by a specific selenoperoxidase, glutathione peroxidase 4 (GPX4) $(1,2)$. We recently identified the substrates and the products of this process as arachidonoyl-phosphatidylethanoamine (AA-PE) and 15-hydroperoxy-AA-PE (15-HOO-AA-PE), respectively $(1,3)$. We further discovered that 2 isoforms of mammalian $15 \mathrm{LOX}-15 \mathrm{LOX} 1$ and $15 \mathrm{LOX} 2$ - form a complex with a scaffold protein, PE-binding protein 1 (PEBP1), that switches the substrate specificity of 15LOX from free AA to AA-PE (3). While 15LOXes are widely represented in many eukaryotic cells (4-6), they were also found in prokaryotes, particu-

Conflict of interest: The authors have declared that no conflict of interest exists Submitted: December 27, 2017; Accepted: July 26, 2018.

Reference information: J Clin Invest. 2018;128(10):4639-4653.

https://doi.org/10.1172/JCI99490. larly in several genera of bacteria $(7,8)$. Surprisingly, $15 \mathrm{LOX}$ was also detected in Pseudomonas aeruginosa (PA1169, hereafter referred to as pLoxA), an organism that lacks oxidizable PUFA-lipid substrates for $15 \operatorname{LOX}(9,10)$. Structural and computational studies established significant differences between the mammalian 15LOXes and pLoxA, including a more spacious catalytic "lobby" readily accommodating a bulky PE in the latter $(11,12)$. Moreover, endogenous non-PUFA-PE was found to cocrystallize with the protein in spite of the fact that it is a very poor substrate for the enzyme (12).

In an attempt to resolve this conundrum, we suggested that, as a bacterial pathogen, $P$. aeruginosa can oxygenate AA-PE in host cells and induce "theft-ferroptosis" by hijacking the mammalian PUFA-PE and death program. Here, we discovered that a biofilm-producing mutant of $P$. aeruginosa is capable of inducing ferroptosis in human bronchial epithelial (HBE) cells via enhanced expression of pLoxA and oxidation of host cell AA-PE to 15-HOOAA-PE. Clinical P. aeruginosa isolates from persistent lower respiratory infection patients caused pLoxA-dependent ferroptosis of HBE cells. By employing global redox phospholipidomics, we further detected elevated levels of 15-HOO-AA-PE in airway tis- 
A

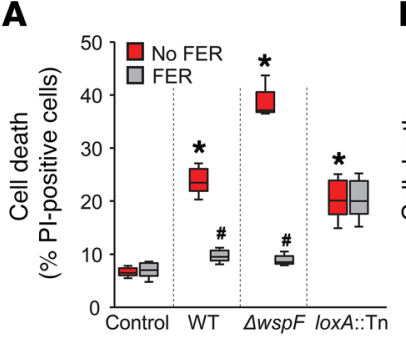

B

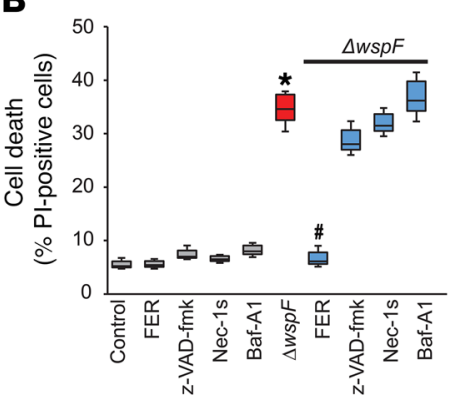

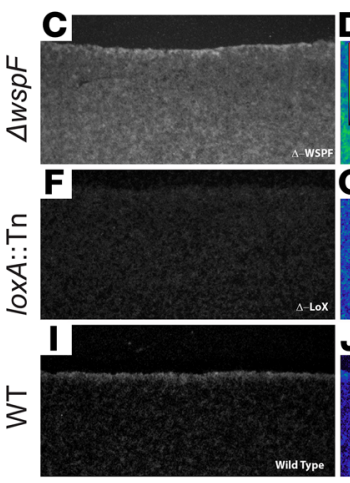
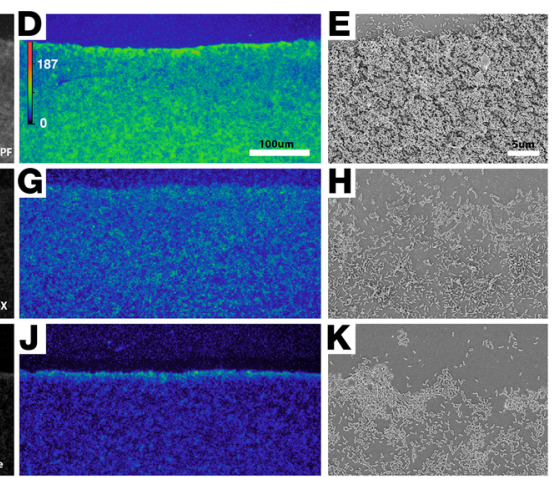

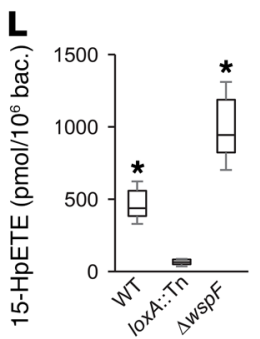

$\mathbf{N}$

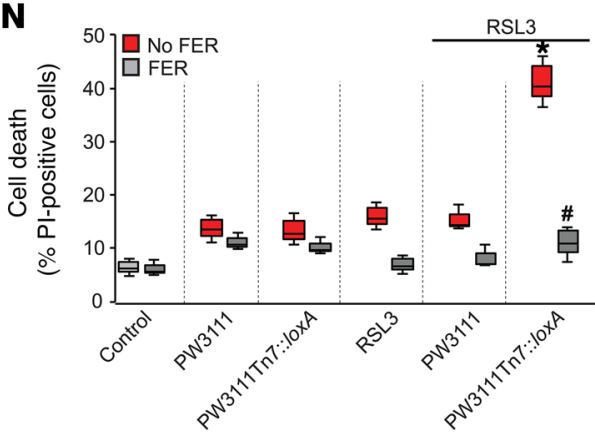

M

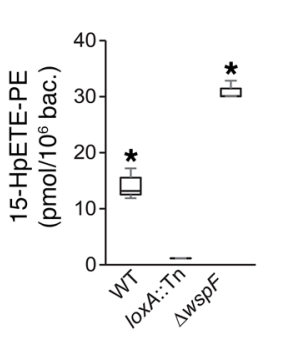

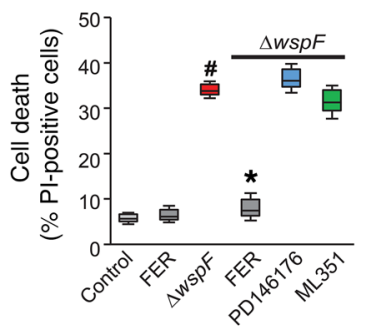

$\mathbf{0}$

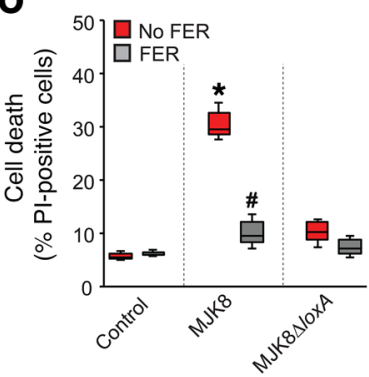

Figure 1. pLoxA is required for $\boldsymbol{P}$. aeruginosa supernatant-induced ferroptosis. (A) HBE cells were treated with supernatants (10 $\mu \mathrm{g}$ each) from WT, $\triangle W s p F$, or pLoxA-deficient mutant loxA::Tn (PW3111) with or without ferrostatin-1 (FER, $0.2 \mu \mathrm{M})$. Cell death (20 hours) assessed by Pl; mean $\pm \mathrm{SD}$, ${ }^{*} P<0.05$ vs. control (untreated HBE cells), $\# P<0.05$ vs. corresponding no FER treatment; $n=3$. (B) $\triangle w s p F$ supernatant alone or with $z$-VAD-fmk (20 $\mu M)$, necrostatin-1s (Nec-1s, $20 \mu \mathrm{M}$ ), bafilomycin-A1 (Baf-A1, $1 \mathrm{nM}$ ). FER, positive control; mean $\pm \mathrm{SD},{ }^{*} P<0.05$ vs. control (untreated), ${ }^{\#} P<0.05 \mathrm{vs} . \Delta \mathrm{ws} p F$ only; $n=3$. (C-K) Representative fixed biofilms on glass coverslips stained with anti-pLoxA antibodies (green) (D, G, and J) or analyzed by SEM (E, H, and $\mathbf{K}$ ) (of 3 performed). (L) Dioxygenase activity: WT, $\triangle W s p F$, or loxA::Tn supernatants were incubated with DOPC/AA (1:10) or DOPC/SAPE (1:1) liposomes (10 minutes, $37^{\circ} \mathrm{C}$ ). AA oxidation product 15-HpETE (left panel) or 15-HpETE-PE from AA-PE (right panel) assessed by LC-MS; normalized to bacteria (bac.)/ml of supernatant; ${ }^{*} P<0.05$ vs. loxA::Tn supernatant; $n=3$. (M) Effect of 15LOX-specific inhibitors (PD146176 and ML351; 1.0 $\mu$ M) on $\Delta$ wspF ferroptosis. RSL3 (200 $\mathrm{nM}$, left panel) was a positive control (both inhibitors were effective against host $15 \mathrm{LOX}$ ); mean $\pm \mathrm{SD},{ }^{\#} P<0.05 \mathrm{vs}$. control (untreated), ${ }^{*} P<0.05$ vs. RSL3 or $\Delta \mathrm{wspF}$ supernatant; $n=3$. (N) Bacterial cell lysates (pLoxA-deficient or complemented, $100 \mu \mathrm{g}$ each) were incubated with SAPE (100 $\mu \mathrm{M}, 30$ minutes) and then added to RSL3-pretreated ( $20 \mathrm{nM})$ HBE cells with or without FER $(0.2 \mu \mathrm{M})$. Mean \pm SD, ${ }^{*} P<0.05$ vs. RSL3, ${ }^{2} P<0.05$ vs. no FER PW3111 Tn7-loxA; $n=3$. (0) HBE cells were incubated with supernatant from MJK8 or its loxA-KO strain (MJK8AloxA) in the presence or absence of FER (0.2 $\mu M)$. Mean \pm SD, ${ }^{*} P<0.05$ vs. control (untreated HBE cells), ${ }^{\#} P<0.05$ vs. no FER MJK8 supernatant; $n=3$. One-way ANOVA for $\mathbf{A}, \mathbf{B}$, and $\mathbf{L}-\mathbf{0}$.

sues from patients with cystic fibrosis (CF) but not from patients with emphysema or CF without $P$. aeruginosa in airway cultures. Assuming that disruption of epithelial barrier and immune-regulatory functions are important for pathogenesis of $P$. aeruginosaassociated respiratory diseases, we propose that pLoxA may represent a new therapeutic target.

\section{Results}

Biofilm-producing prokaryote P. aeruginosa utilizes pLoxA to induce ferroptotic death in HBE cells. Transition of $P$. aeruginos a from planktonic to biofilm growth is associated with an increase in expression of its lipoxygenase (ploxA) gene (13). Given the recent discovery of the leading role of human lipoxygenases (15LOX1 and 15LOX2) in triggering the ferroptotic cell death program (3), we asked whether $P$. aeruginosa can induce ferroptosis in host HBE cells. Like many Gram-negative bacteria, $P$. aeruginosa produces and releases into the environment membrane vesicles containing many intracellular proteins, including pLoxA $(14,15)$. These vesicles could be isolated as supernatants after centrifugation of growing cultures. Given the high prevalence of inactivating mutations of the wspF gene in CF clinical isolates and its hyper-biofilm nature (16-18), we tested supernatant from the $\Delta w s p F$ mutant of $P$. aeruginosa grown in biofilm conditions as an inducer of ferroptosis in HBE target cells. Supernatants from the $\triangle w s p F$ mutant were significantly more efficient in inducing cell death preventable by a specific inhibitor of ferroptosis, ferrostatin-1, than WT supernatants (Figure 1A). Nota- 
bly, the planktonic supernatants did not induce this type of cell death (Supplemental Figure 1A; supplemental material available online with this article; https://doi.org/10.1172/JCI99490DS1). By employing several commonly used inhibitors of alternative cell death programs - z-VAD-fmk (for apoptosis) (19), necrostatin-1s (for necroptosis) (20), and bafilomycin-A1 (for autophagic death) (21) - we investigated the nature of the death pathway triggered by the $\Delta w s p F$ mutant (Figure 1B). In contrast to ferrostatin-1, none of the tested inhibitors revealed significant suppression of cell death induced by $\triangle w s p F$ supernatant as evidenced by propidium iodide (PI), MTT, and PI/annexin V double staining assessments (Figure 1B and Supplemental Figure 1, B-D).

To test the involvement of pLoxA in ferroptotic death, we analyzed the level of the protein in lysates of WT, $\triangle w s p F$, and loxA::Tn (PW3111) P. aeruginosa. As expected, $\triangle w s p F$ mutant contained markedly higher levels of pLoxA than the WT strain (Supplemental Figure 2A). Similarly, larger amounts of pLoxA were found in biofilms formed by the $\Delta w s p F$ mutant than by the WT strain (Figure $1, \mathrm{C}-\mathrm{K})$. No detectable signals were found in lysates of a pLoxAdeficient loxA::Tn (PA1169 transposon deletion mutant, PW3111) strain, which did not affect abiotic biofilm formation compared with the WT parent strain (13) (scanning electron microscopy [SEM] images, Figure 1, $\mathrm{H}$ and $\mathrm{K}$, and Supplemental Figure 2B). To assess oxygenase enzymatic activity, we performed liquid chromatography-mass spectrometry-based (LC-MS-based) analysis of the products generated by WT, $\triangle w s p F$ mutant, and loxA::Tn mutant (Figure 1L). We employed 2 substrates, free AA (Figure 1L, left panel, and Supplemental Figure 2C) and AA esterified into PE (AA-PE) (Figure 1L, right panel, and Supplemental Figure 2D), and determined that the oxygenation activity of the $\triangle w s p F$ mutant was more than an order of magnitude greater than that of the loxA::Tn mutant. Given that the mutant devoid of pLoxA induced necroptotic but not ferroptotic cell death (Figure 1A and Supplemental Figure 2E), we concluded that the presence of pLoxA is indispensable for generating pro-ferroptotic signals.

To examine whether exogenous pLoxA in P. aeruginosa supernatants is the major contributor to ferroptosis in HBE cells, we studied the effects of 2 specific small molecule inhibitors of mammalian 15LOX, PD146176 and ML351 $(19,22)$. In agreement with previous reports (1), ferroptosis induced in HBE cells by an inhibitor of GPX4, RSL3, was markedly suppressed by both inhibitors (Figure 1M, left panel). In contrast, both mammalian 15LOX-specific inhibitors, which do not target pLoxA, were ineffective in protecting against $P$. aeruginosa-induced ferroptosis (Figure $1 \mathrm{M}$, right panel). However, baicalein, a nonspecific lipoxygenase inhibitor, suppressed cell death induced by both RSL3 and P. aeruginosa (Supplemental Figure 2F).

To further scrutinize the role of pLoxA in ferroptosis, we used 2 genetic approaches. First, we generated a loxA::Tn complemented strain (PW3111 Tn7::loxA) expressing pLoxA. Bacterial cell lysates prepared from complemented PW3111 Tn7::loxA and pLoxA-deficient loxA::Tn (PW3111) strains grown in biofilm conditions were incubated with the pLoxA substrate stearoylarachidonoyl-PE (SA/AA-PE) to generate the pro-ferroptotic signal 15-HOO-SA/AA-PE. We added this reaction mixture to HBE cells pretreated with RSL3 in the presence or absence of ferrostatin-1. Cell lysate from pLoxA expressing complemented strain induced cell death that was rescued by ferrostatin-1, while lysate from the pLoxA-deficient strain (loxA::Tn) did not induce ferroptosis (Figure $1 \mathrm{~N}$ and Supplemental Figure 2, G and $\mathrm{H}$ ). Second we used a PAO1 isogenic strain, rugose small colony variant MJK8, and its loxA-deleted strain, MJK8DPA1169 (16) (herein referred to as MJK8 $\Delta$ loxA). Supernatants from the MJK8 strain induced cell death prevented by ferrostatin-1, while the MJK8 $\Delta$ loxA supernatant did not induce ferroptotic cell death of target HBE cells (Figure 10 and Supplemental Figure 2I).

pLoxA-generated 15-HOO-AA-PE act as pro-ferroptotic signals in $H B E$ cells. To interrogate the role of lipid hydroperoxides as pro-ferroptotic signals, we employed a fluorogenic probe, Liperfluo, which, like GPX4, selectively reduces hydroperoxy lipids to respective alcohols to yield a fluorescent product $(23,24)$. Incubation with the $\triangle w s p F$ mutant supernatant induced a time-dependent increase in the fluorescent signal in HBE cells, which was fully protected by ferrostatin-1 (Figure 2A). This response was similar to the one elicited by the treatment of HBE cells with the GPX4 inhibitor RSL3 as a positive control (1). Notably, Liperfluo fluorescence originated in a single cell, and over time was spreading and detectable in clusters of many surrounding cells (i.e., in a non-cell-autonomous fashion) (Figure 2B). Assuming that Liperfluo fluorescence responses report the early generation and propagation of pro-ferroptotic LPO products, we monitored the dissemination of cell death using SYTOX green labeling of HBEs. Similar to previously reported results (2527), $P$. aeruginosa $\triangle w s p F$ supernatant-induced cell death was also associated with the appearance of clusters of dying cells (Supplemental Figure 3). As expected, this clonal loss of plasma membrane integrity revealed by SYTOX green occurred at later time points than Liperfluo positivity of cells.

To determine the molecular identity of lipid oxidation products generated by $P$. aeruginosa $\triangle w s p F$ supernatant in HBE cells, we performed quantitative phospholipidomics analysis (Figure 2, $\mathrm{C}-\mathrm{E}$ ). We established that PE contained 15 oxidized species (Figure 2D and Supplemental Table 1). Importantly, treatment of HBE cells with ferrostatin-1 selectively reduced the levels of 4 oxidized $\mathrm{PE}$ (PEox) species (Figure 2E) that we identified as PE containing either 15-HOO-AA-PE or 12-HOO-AA-PE in the $s n-2$ position (Supplemental Figures 4 and 5) and were previously reported as predictive biomarkers of RSL3-induced ferroptosis (1).

To further explore the role of phospho-LPO in P. aeruginosainduced death, we manipulated the content and molecular speciation of lipids in target cells. Our previous work showed that AA supplementation sensitizes cells to ferroptosis via increased synthesis of AA-PE as a pro-ferroptotic oxidation substrate for $15 \mathrm{LOX}$ (1). Exogenous AA significantly enhanced ferroptosis induced by WT and $\triangle w s p F$ supernatants but had no effect on pLoxA-deficient loxA::Tn (PW3111) supernatant (Figure 3A).

Additional testing was performed by genetic manipulations of PL biosynthetic (acyl-CoA synthetase long-chain family member 4 [ACSL4]) or remodeling (lysophosphatidylcholine acyltransferase 3 [LPCAT3]) pathways. Knockdown (KD) in HBE cells of ACSL4, which catalyzes the formation of long-chain PUFA-CoA (AA-CoA) (28), completely prevented $P$. aeruginosa-induced ferroptosis (Figure 3B). Similarly, KD of LPCAT3, involved in reacylation (Lands cycle) of lysophospholipids (29), also markedly suppressed ferroptosis in HBE cells (Figure 3B and Supplemen- 
A
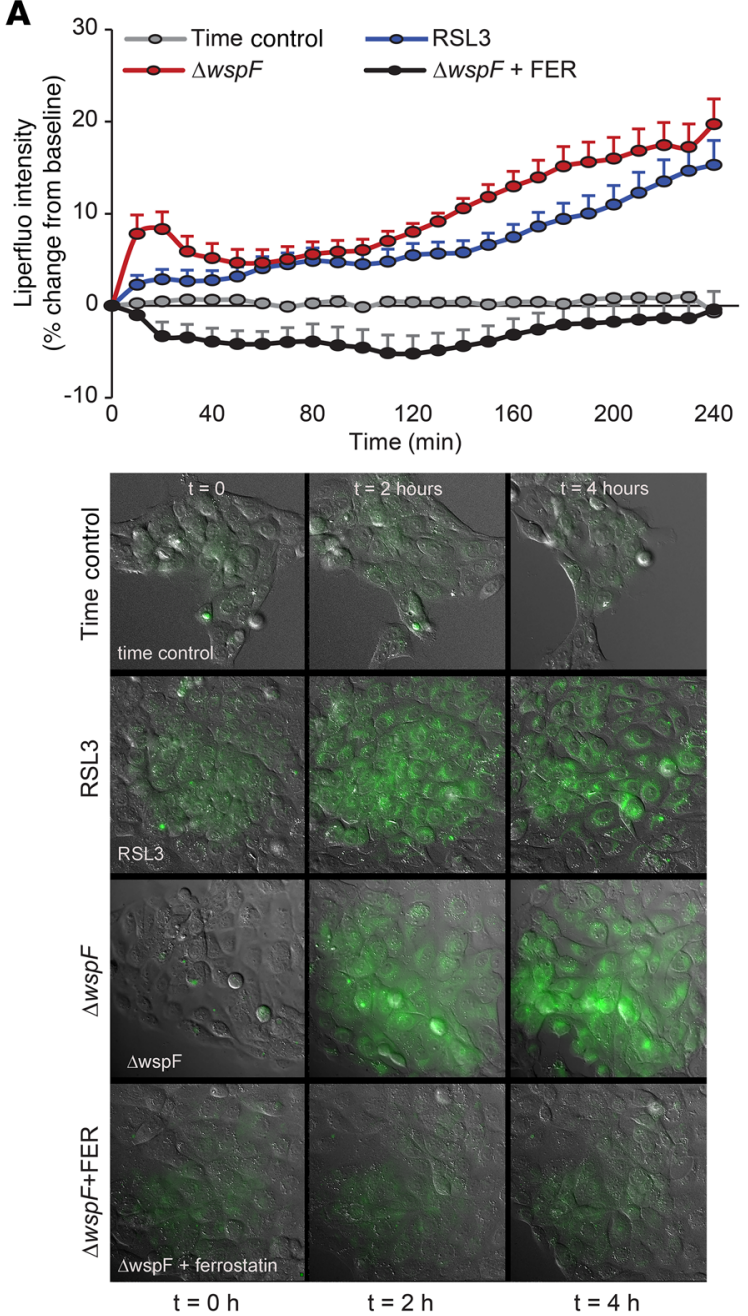

B

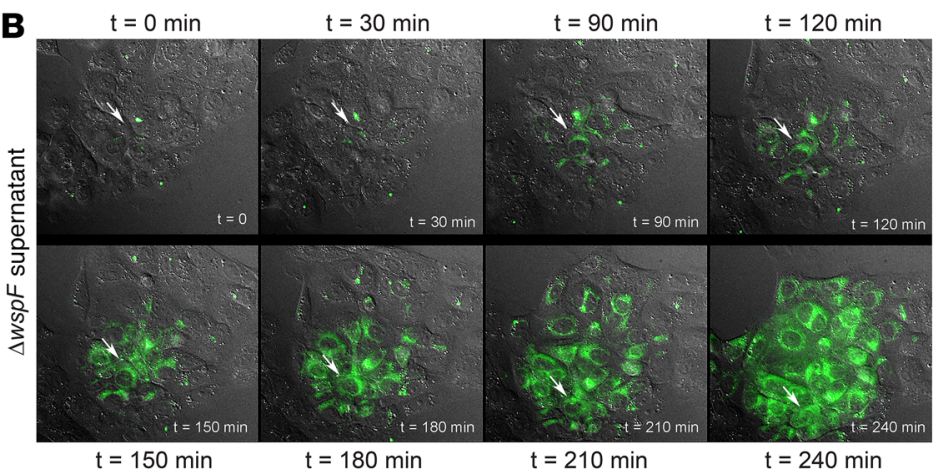

C

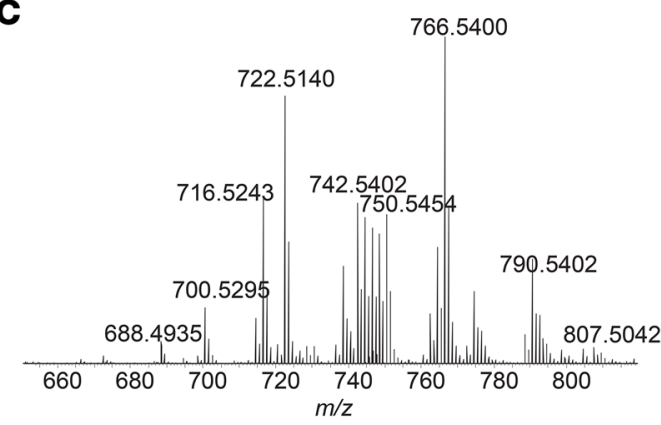

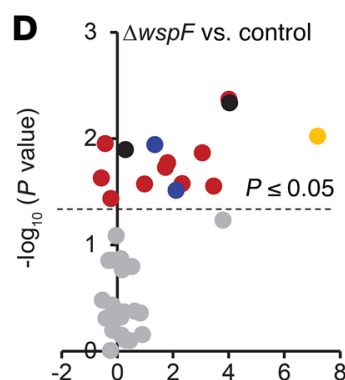

$\log _{2}$ (fold change)

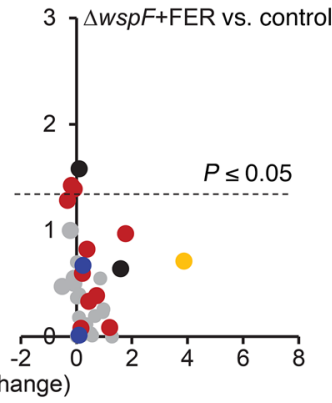

PE-38:5+2[O]
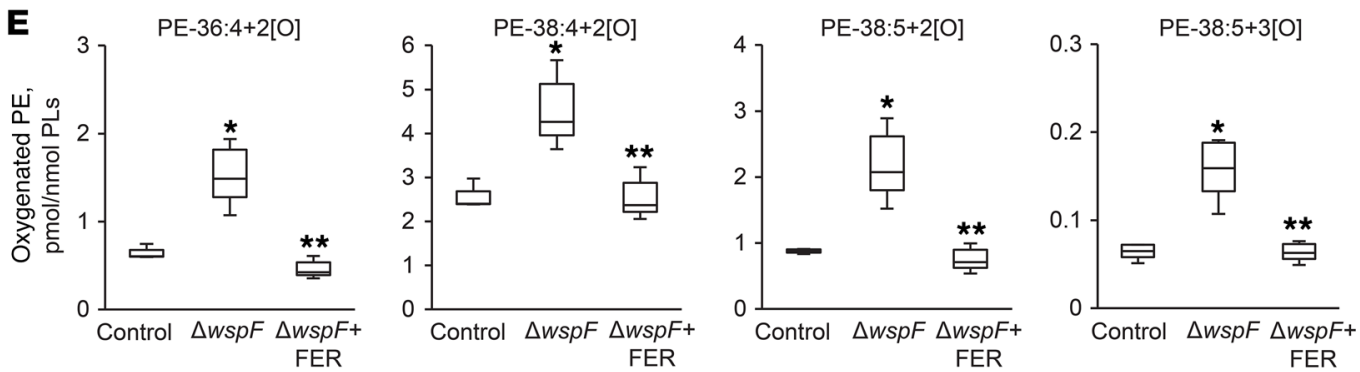

Figure 2. P. aeruginosa supernatants generate pro-ferroptotic hydroperoxy lipid signals in HBE cells. (A) Detection of lipid hydroperoxides by live cell fluorescence imaging of Liperfluo in HBE cells treated with RSL3 (200 nM) or $\Delta w s p F$ supernatant for 4 hours. Top panel shows time course of changes in the fluorescence intensity from baseline. Bottom panel shows typical changes in fluorescence (of 4 performed) in one stage position (of 10$)$ at 3 time points ( 0,2 , and 4 hours). Time control used as a negative control and RSL3 as a positive control for ferroptosis. For statistical analysis, each stage position was counted as one data entry. (B) Typical Liperfluo response (of 4 performed) to $\triangle \mathrm{wspF}$ showing synchronized spreading of the oxidized lipid signal within cells. The seed point for the signaling is indicated by an arrow in the $t=0$ image; the subsequent time course shows that the signals expanded in a clonal way to include multiple surrounding cells. (C) Typical mass spectrum of PE molecular species in lipid extracts from HBE cells (of 3 performed). (D) Volcano plots of $\triangle w s p F$ biofilm supernatant-induced changes in the levels of oxygenated PEs $\left[\log _{2}\right.$ (fold change)] versus significance $\left[-\log _{10}(P\right.$ value)] by $t$ test in HBE cells in the absence (left panel) and presence (right panel) of ferrostatin-1 ( $0.2 \mu \mathrm{M})$. Yellow, red, blue, and black circles represent PE plus 1, 2, 3, and 4 oxygens, respectively. $n=3$. (E) Quantitative assessments of 4 PEox species (previously identified as pro-ferroptotic signals) generated in HBE cells exposed to $\Delta$ wspF supernatants. ${ }^{*} P<0.05$ vs. control, ${ }^{* *} P<0.05$ vs. $\triangle$ wspF (1-way ANOVA); $n=3$.

tal Figure 6A). Intriguingly, pLoxA-dependent biofilm formation (13) and epithelial cell death were interconnected, as inhibition of ferroptosis by ferrostatin- 1 was accompanied by a 2 -fold growth decrease in a biotic model (Figure 3C).
GPX4 can directly reduce hydroperoxy-PLs to non-pro-ferroptotic hydroxy-PLs $(24,30,31)$. Depletion of GPX4 - critical for the development of ferroptotic response - has been observed in a variety of cells treated with RSL3 $(1,31,32)$. P. aeruginosa $\triangle w s p F$ supernatant 

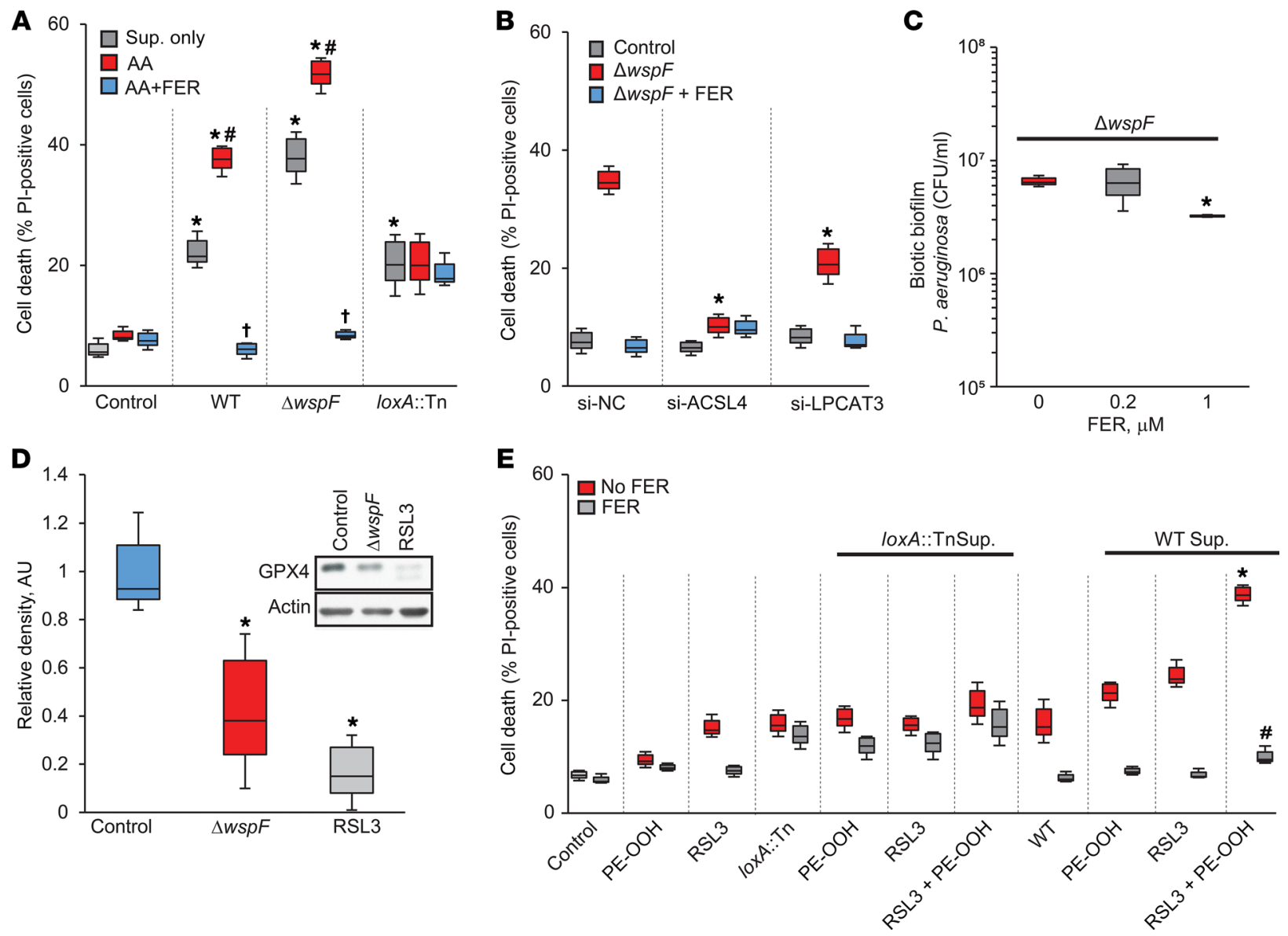

Figure 3. P. aeruginosa-induced ferroptosis is affected by manipulations of PLs in HBE cells. (A) Exogenous AA enhances $P$. aeruginosa-induced ferroptosis. HBE cells were treated with WT, $\triangle w s p F$, or loxA::Tn supernatant (Sup.) alone or in combination with AA (2.5 $\mu \mathrm{M})$ for 20 hours at $37^{\circ} \mathrm{C}$. Cell death was estimated as mentioned above. Data are presented as mean $\pm \mathrm{SD},{ }^{*} P<0.05 \mathrm{vs}$. control (untreated), ${ }^{*} P<0.05$ vs. corresponding supernatant only, ${ }^{\dagger} P$ $<0.05$ vs. corresponding AA treatment; $n=3$. (B) HBE cells were transfected with scrambled siRNA (si-NC) or siRNA against ACSL4 or LPCAT3. Transfected cells were treated with $\triangle w s p F$ supernatants with or without ferrostatin- $1(0.2 \mu \mathrm{M})$ for 20 hours at $37^{\circ} \mathrm{C}$ before estimating cell death. Data are presented as mean $\pm \mathrm{SD} ;{ }^{*} P<0.05$ vs. si-NC $\Delta w s p F ; n=3$. (C) Ferrostatin-1 inhibits $P$. aeruginosa-induced biofilm formation on HBE cells. Polarized HBE cells were incubated with $\triangle W s p F P$. aeruginosa (MOI of 25 ) for 1 hour. After washing unattached bacteria, HBE cells were cultured in the absence or presence of ferrostatin-1 $(0.2$ and $1.0 \mu \mathrm{M})$ for 5 hours. Biofilms were removed and lysed with Triton $X-100(0.1 \%)$, and the number of bacteria in each sample was determined by CFU assay. Data are presented as mean \pm SD, ${ }^{*} P<0.05$ vs. $\Delta w s p F$ with no FER; $n=3$. (D) Treatment of HBE cells with $\Delta w s p F$ supernatant decreases the content of GPX4. Cells were treated with $\triangle w s p F$ supernatant and incubated for 20 hours at $37^{\circ} \mathrm{C}$. Samples were collected and processed for Western blotting to determine the levels of GPX4. RSL3, a covalent GPX4 inhibitor, was used as a positive control. For quantification, the band intensity of GPX4 protein was normalized to respective band intensity of actin. Inset: typical Western blot (of 3 performed). Data are presented as mean $\pm S D$, ${ }^{*} P<0.05$ vs. control (untreated) HBE cells; $n=3$. (E) Exogenous 15-HOO-AA-PE elevated WT but not loxA-deficient loxA::Tn supernatant-induced cell death. HBE cells pretreated with RSL3 (20 nM for 4 hours) were incubated with PE-OOH alone or with supernatant from loxA::Tn or WT in the presence or absence of ferrostatin-1 $(0.2 \mu \mathrm{M})$. Data are presented as mean $\pm \mathrm{SD},{ }^{*} P<0.05$ vs. control (untreated) HBE cells, ${ }^{\#} P<0.05$ vs. FER; $n=3$. One-way ANOVA.

induced substantial loss of GPX4 protein in HBE cells (Figure 3D), thus eliminating this anti-ferroptotic regulator. Due to the reduction of 15-HOO-AA-PE to 15-HO-AA-PE by endogenous GPX4 in HBE cells, recombinant pLoxA was unable to induce ferroptotic cell death (Supplemental Figure 6B), even though it generated 15-HOO-AAPE in a model system (Supplemental Figure 6C). However, inactivation of GPX4 by RSL3 facilitated ferroptosis induced by WT but not pLoxA-deficient loxA::Tn supernatant in 15-HOO-AA-PE-sensitized HBE cells, thus signifying the role of pLoxA (Figure 3E).

Computational analysis identifies evolutionarily conserved specific features of $p L o x A$ required for AA-PE oxidation. Lipoxygenases are found in different domains of life, where their activities are associated with vitally important functions such as differentia- tion, proliferation, and contractility $(4,5)$. Mammalian and bacterial lipoxygenases show little sequence identity. Alignment of the pLoxA sequence against the structurally resolved mammalian sequences (15LOX1 human and 15LOX2 human) (Supplemental Figure 7A) yielded respective fractional sequence identities of 0.25 and 0.31 (note that 15LOX1 and 15LOX2 themselves also share only $36 \%$ sequence identity). Phylogenetic analysis of Pfam (33) family members revealed that bacterial LOXes diverged from those of animals and plants at approximately the same time as the animal and plant lipoxygenases diverged from each other (Figure 4A, left panel). The bacterial LOXes, however, seem to be more similar to animal and plant lipoxygenases than to lipoxygenases from other eukaryotes (e.g., fungi), suggesting that there might 
A

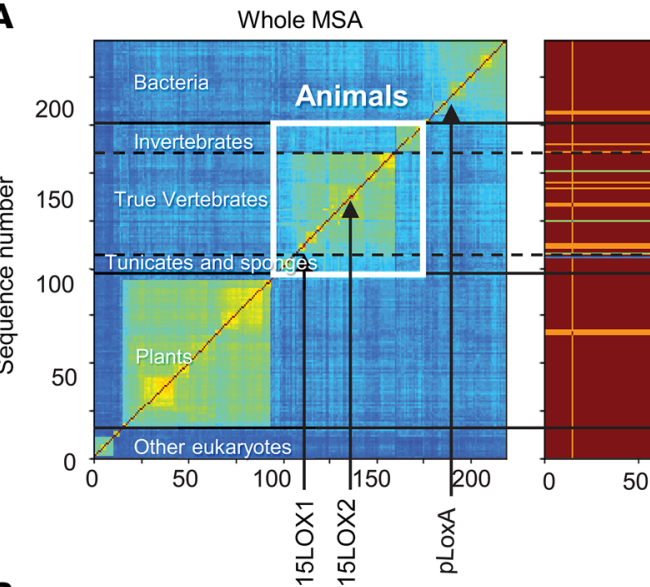

Catalytic site
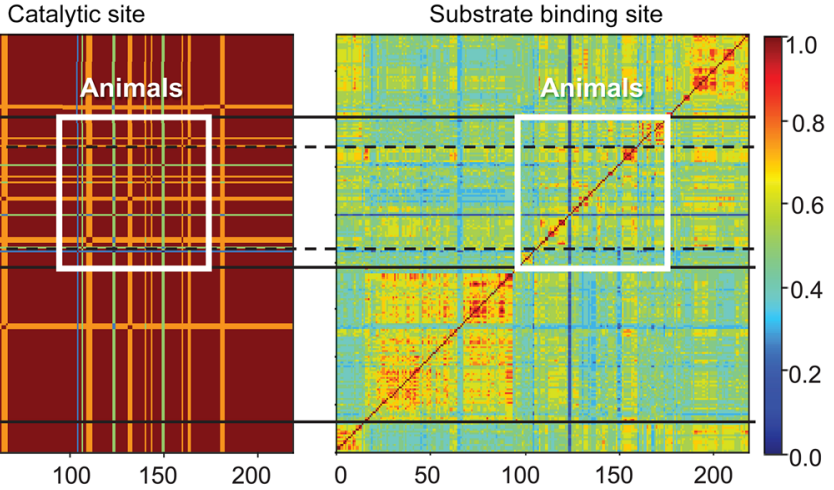

B
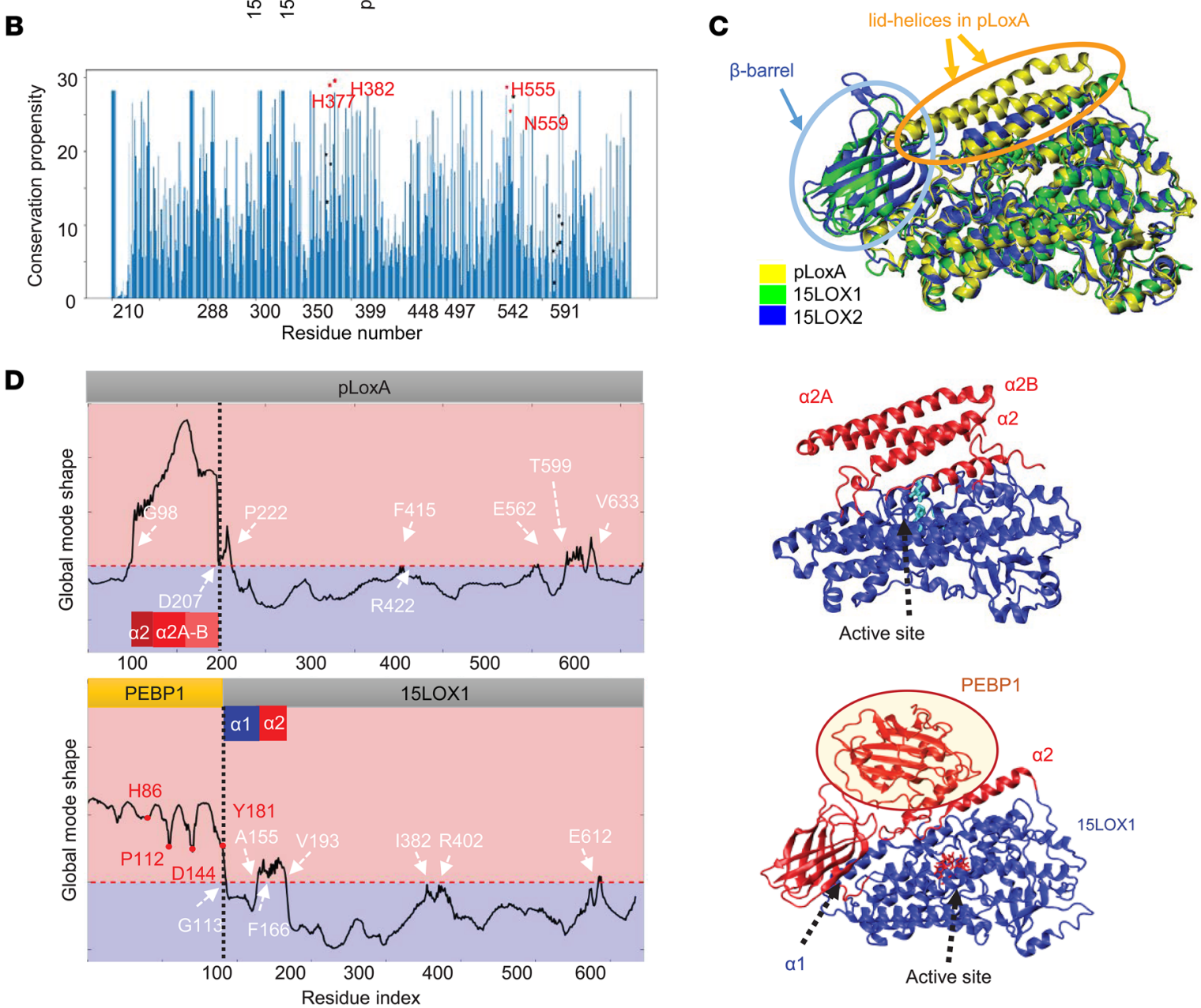

Figure 4. Evolutionary analysis and computational modeling of pLoxA sequence and structure. (A) Comparing sequences of LOX homologs. The panels show sequence identity matrices obtained from 218 LOX sequences from all domains of life. High sequence similarity is in red, intermediate in yellow/green, and low in blue. Matrix generated for the entire LOX sequence (left panel) shows blocks evident for plants, animals, and bacteria, indicating their divergence. Light blue shading between these blocks indicates a degree of sequence similarity higher than to the block of other eukaryotes at the bottom (dark blue off-diagonal). High conservation for the catalytic site is shown in the middle panel. Residues involved in substrate recognition (right panel) exhibit a moderate level of conservation and species-specific differentiation, evidenced by the blocks for plants, animals, and bacteria. (B) Conservation propensity of LOX. The ordinate displays the Shannon entropy, subtracted from maximum entropy, plotted as a function of residue index; the peaks represent the most conserved residues. Note that catalytic residues (labeled) are among them. Residues involved in ligand coordination are indicated by the black dots. (C) Structural comparison of pLoXA and mammalian LOXes - 15LOX1 and 15LOX2. The structures resolved for pLoXA (yellow ribbon), 15LOX1 (green), and 15LOX2 (blue) are superimposed and display the missing $\beta$-barrel of the bacterial pLoxA and its additional $\alpha$-helical hairpin motif at the C-terminus. (D) Comparison of the global motions of pLoxA and 15LOX1 complexed with PEBP1. Displacements of residues along the global mode axis obtained from GNM analysis of pLoxA (left, top) and 15LOX1-PEBP1 complex (left, bottom) dividing the structure into 2 domains that exhibit anticorrelated motion (above and below the dashed line). Residues at the crossover region (hinge centers or anchors) are labeled. The anticorrelated groups of residues are shown in red or blue (right, top and bottom). 
A

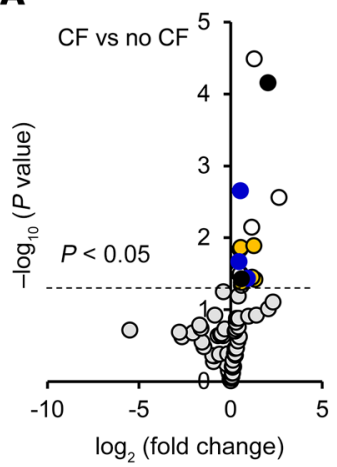

B

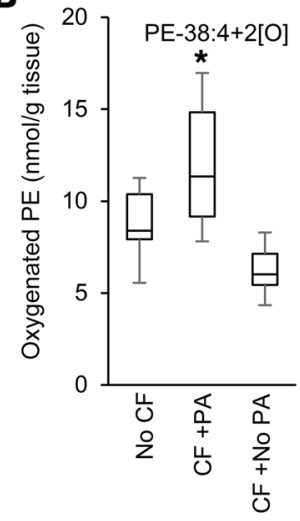

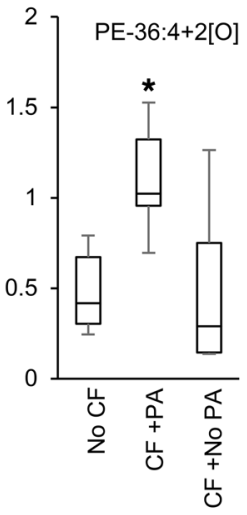

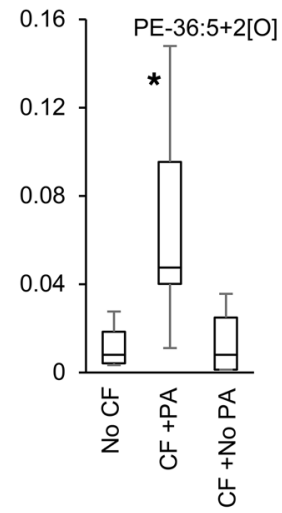

C

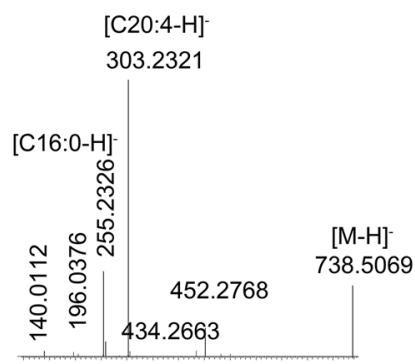

100200300400500600700 $m / z$

D

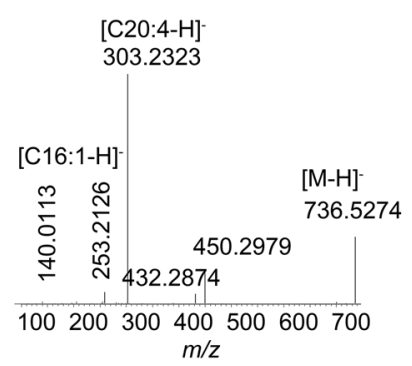

$\mathbf{E}$
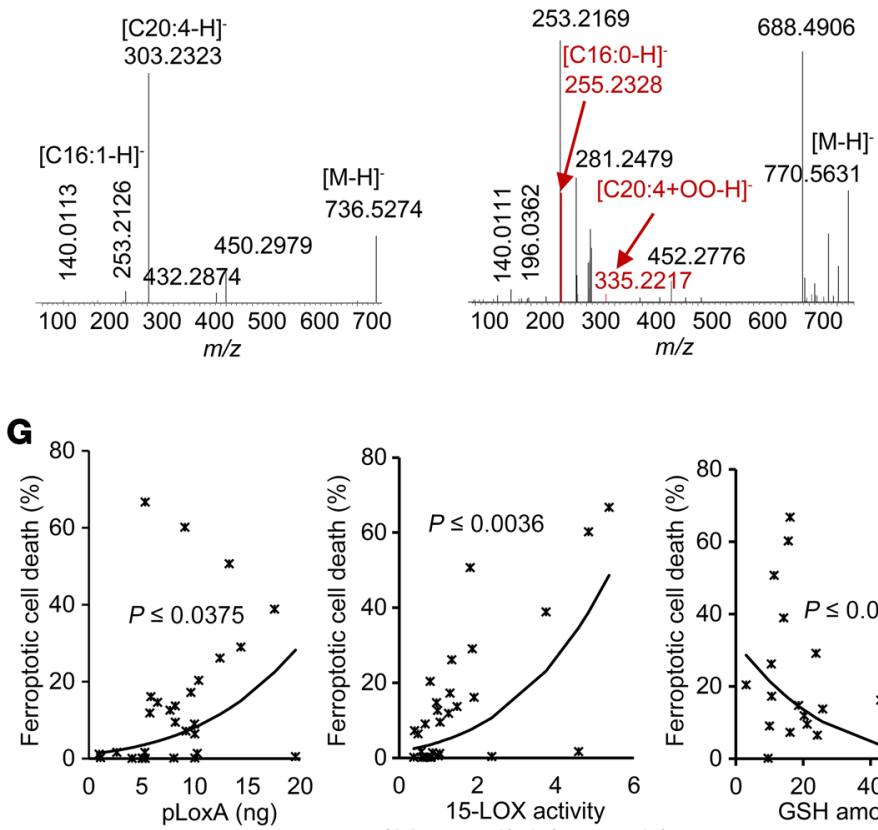
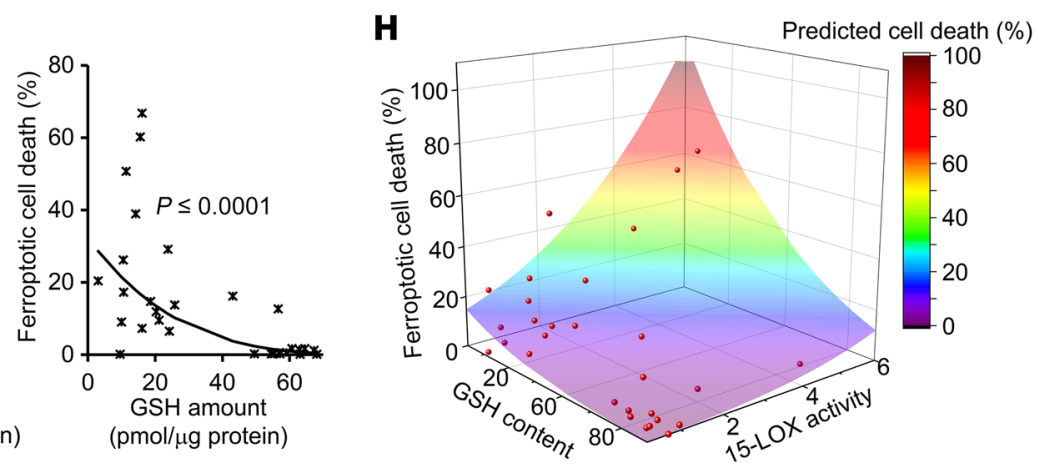

Figure 5. Redox phospholipidomics reveals pro-ferroptotic oxygenated PE species in airway tissue samples from patients with CF. (A) Volcano plot of differences in the levels of PEox species in CF vs. non-CF patients $-\log _{2}$ (fold difference) vs. significance $\left[-\log _{10}(P\right.$ value)]. Airway tissues from patients with emphysema were used as controls. Yellow, red, blue, and black circles represent PEox species containing 1, 2, 3, and 4 oxygens, respectively. $n=10$ (B) Quantitative assessments of PEox species. Non-CF samples $(n=10)$, CF samples with $P$. aeruginosa $(P A ; n=6)$, CF samples with other bacterial pathogens (B. cenocepacia or $A$. xylosoxidans and M. abscessus, $n=4)$. (C-E) MS/MS-based identification of PEox molecular species in samples from patients with CF. Typical MS/MS spectra of PEox precursors with $m / z$ 738.5 (PE16:0/20:4) (C) and 736.5 (PE16:1/20:4) (D) containing AA (C20:4 at $m / z$ 303.2) at sn-2 positions. MS/MS spectra of PEox with $m / z$ 770.5 (PE16:0/20:4+20) containing oxygenated AA (C20:4+20 at $m / z$ 335.2) (E) formed from the PE species with $\mathrm{m} / \mathrm{z} 738.5$ plus 2 oxygens (of 3 performed). (F) Tobramycin-resistant $P$. aeruginosa clinical isolates (TRPA002-TRPA122) from patients with persistent lower respiratory infection induce ferroptosis. Biofilm supernatants from $P$. aeruginosa isolates incubated with HBE cells with or without ferrostatin-1 $(0.2 \mu \mathrm{M})$. Ferroptosis was calculated by subtracting from cell death induced by supernatants the cell death in the presence of ferrostatin-1. Ferroptotic activity of clinical isolates correlated significantly with pLoxA amounts (determined by Western blotting; $n=29)(\mathbf{G}$, left panel), displayed strong positive dependence on 15LOX activity (G, middle panel) $(P \leq 0.0036)$, and correlated negatively with the GSH level of HBE cells after treatment $(\mathbf{G}$, right panel). (H) $3 \mathrm{D}$ plot illustrating jointly predicted ferroptotic activity of 15LOX activity and GSH $(n=29) . n$ is number of samples. One-way ANOVA.

have been a gene transfer event since the prokaryote/eukaryote split and the development of multicellular organisms (Figure 4A, left panel). This is further illustrated by the phylogenetic tree, which shows clustering of sequences from particular organisms. Bacteria are represented throughout the tree, although there is a main group that includes pLoxA (Supplemental Figure 7B). Alignment of the conserved structural core of pLoxA revealed 223 bacterial sequences (of $\sim 30,000$ examined) sharing a sequence identity of $20 \%$ or more, and of these, 37 had greater than $55 \%$ sequence identity with pLoxA. Supplemental Figure 8 shows their pairwise sequence identities (Supplemental Figure 8A) and the corresponding phylogenetic tree (Supplemental Figure 8B). The tree reveals discrete clusters of pathogenic bacteria, including a large cluster that groups Pseudomonas (green) in close proximity to Burkholderia (blue), in accordance with a previous analysis (7). Further examination of the location of conserved residues on the 
LOX structure confirmed the tendency of core residues, near the catalytic site, to be conserved, whereas peripheral regions, including a helical hairpin that distinguishes $P$. aeruginos a from mammalian 15LOX1 and 15LOX2, exhibited significant sequence dissimilarity (or specificity) (Supplemental Figure 8C).

Similar to other LOX enzymes, pLoxA employs non-hemecoordinated $\mathrm{Fe}$ for dioxygenase catalysis (12). Therefore, we assessed the level of conservation of LOX amino acids, with peaks indicating the most conserved residues (Figure 4B). As expected, the catalytic site residues (H377, H382, H555, and N559 in pLoxA) were noted among the peaks. Examination of their evolutionary patterns across species confirmed their high level of conservation (Figure 4A, middle panel), whereas residues involved in substrate recognition exhibited a moderate level of conservation, with species-specific evolutionary trends (Figure 4A, right panel).

As an opportunistic bacterial pathogen, $P$. aeruginosa has acquired multiple adaptive genes favoring the highly adaptive phenotypes required to thrive and outcompete many other species in the highly proinflammatory environment of the host (34-36). Among them is expression of pLoxA that facilitates the transition from a planktonic to biofilm-forming state in the presence of the airway epithelium (13). Utilization of secreted pLoxA to execute theft-ferroptosis via generation of $15-\mathrm{HOO}-\mathrm{AA}-\mathrm{PE}$ signals not only destroys the epithelial barrier but may also compromise immune-stimulating functions. To fulfill these important functions, P. aeruginosa has developed an enzyme with structural features distinct from mammalian (human) 15LOX1 (37) and 15LOX2 (38) (Figure 4C). pLoxA has $2 \mathrm{C}$-terminal $\alpha$-helical hairpins, composed of helices $\alpha 2 \mathrm{a}$ and $\alpha 2 b$, which are absent in the mammalian 15LOXs. The C-terminal helical hairpin modifies the organization of a ligand-binding pocket near the catalytic site favoring the binding of bulky substrates such as AA-PE (12). On the other hand, it lacks the N-terminal $\beta$-barrel domain typical of mammalian and human 15LOXes (37). The remaining portions of the structure are closely superimposable between pLoxA and mammalian LOXes (Figure 4C). Dynamic motions of the $2 \alpha$-helices modify the organization of the catalytic site, favoring the binding of bulkier substrates, such as AA-PE, versus free AA in pLoxA (Supplemental Figure 9A). Gaussian network model (GNM) analysis of pLoxA unveiled an additional regulatory mechanism whereby these helices act as a lid, regulating closed and opened states of the catalytic site for substrate binding (Figure 4D, upper panels; Supplemental Video 1). The mechanical role played by this extra lid is that it recruits the adjacent $\alpha 2$ helix of pLoxA to enable a local hinge bending mechanism, which in turn facilitates the binding of PE-esterified AA to a site (Supplemental Figure 9, A and B) in the vicinity of the catalytic site, just like PEBP1 in the PEBP1-15LOX complex (Figure 4D, lower panels).

Redox phospholipidomics reveals elevated levels of 15-HOO-AA$P E$ in airway tissues of $P$. aeruginosa-infected patients with CF. Chronic biofilm-based infections of the airways with $P$. aeruginosa occur in $70 \%-80 \%$ of patients with CF, representing the leading cause of morbidity and mortality in these patients $(39,40)$. Assuming that there is a $P$. aeruginosa-associated pathogenic mechanism involving pLoxA as virulence factor that induces ferroptotic cell death by regulating host-pathogen interactions at the airway mucosal surface, we performed quantitative redox phospholipidomics studies of airway samples from CF and non-CF patients (Supplemental
Figure 10A). Several oxygenated PE species were elevated in CF versus control samples (Figure 5A and Supplemental Table 2). We identified 3 PEox species whose levels were significantly higher in $\mathrm{CF}$ patients versus non-CF controls (Figure $5 \mathrm{~B}$ ). MS/MS fragmentation analysis identified $\mathrm{PE}(36: 4)$ (Figure 5C) and $\mathrm{PE}(36: 5)$ (Figure 5D) as precursors of the PE oxidation products (Figure 5E). These PEox species were also present in $P$. aeruginosa-treated HBE cells (Figure 2E) and have been reported as predictive pro-ferroptotic biomarkers (1). Microbiological testing revealed that of 10 CF samples, 6 were infected with $P$. aeruginosa (Supplemental Table 3). Remarkably, the content of these 3 PEox pro-ferroptotic signals was specifically elevated in $P$. aeruginosa-infected CF lung samples but not in the samples infected with other pathogens such as Burkholderia cenocepacia, Achromobacter xylosoxidans, or Mycobacterium abscessus (Figure 5B and Supplemental Table 3).

High prevalence of pLoxA in ferroptosis-inducing clinical isolates of $P$. aeruginos a from patients with persistent lower respiratory tract infection. $P$. aeruginosa is one of the leading opportunistic pathogens that causes health care-associated lower respiratory tract infections including ventilator-associated pneumonia (VAP) (41). Lower respiratory tract infections disproportionately affect the elderly, the immunocompromised, and critically ill patients. High mortality rates associated with infections of the lower respiratory tract are further enhanced by increasing numbers of multidrug and extensively drug-resistant strains of $P$. aeruginosa, which lead to treatment failures and persistence of infection $(42,43)$. To assess the significance of pLoxA in persistent lower respiratory tract infection, we examined the effectiveness of tobramycin-resistant $P$. aeruginosa respiratory isolates from 29 patients in an intensive care unit (ICU) to trigger ferroptosis in HBE cells (Figure 5F). We found that the ferroptotic cell death of $P$. aeruginosa clinical isolates correlated positively with the amounts of pLoxA (Figure 5G, left panel, and Supplemental Figure 10B) and pLoxA enzymatic activity, particularly 12,15LOX activity (Figure 5G, middle panel) and not significantly with 5LOX activity (Supplemental Figure 10C). These data are compatible with our findings that 12- and 15-HOO-AA$\mathrm{PE}$ dominate among the PE oxidation products generated by the $\triangle w s p F$ mutant $P$. aeruginosa supernatants in HBE cells (Supplemental Figures 4 and 5). We also found a strong negative correlation between the amounts of glutathione (GSH) in HBE cells (incubated with clinical isolate supernatants for 20 hours) and percent of ferroptotic cell death induced by clinical isolates $(P \leq 0.0001)$ (Figure $5 \mathrm{G}$, right panel). This is compatible with the important role of GSH as the source of reducing equivalents for the GPX4-catalyzed reaction of elimination of pro-ferroptotic $15-\mathrm{HOO}-\mathrm{AA}-\mathrm{PE}$ (via their reduction to $15-\mathrm{HO}-\mathrm{AA}-\mathrm{PE}$ ). Further, assessment of pyocyanin in the isolates revealed no correlation between its content and the effectiveness in causing ferroptosis in HBE cells (Supplemental Figure 10D). Finally, we used the stepwise variable selection method to generate a multivariate model, and we found that the $15 \mathrm{LOX}$ activity of pLoxA and the amount of GSH in HBE cells jointly predicted ferroptotic cell death, with an $R$ of 0.831 (Figure $5 \mathrm{H}$ ).

\section{Discussion}

Execution of the ferroptotic death program has been demonstrated in multiple normal and cancerous mammalian cells and different tissues, where it has been implicated in the pathogenesis 
of chronic and acute diseases such ischemia-reperfusion of liver (44), acute kidney injury (45), and Parkinson's disease (46). Ferroptosis has been also documented in plants (47). Here we report, for the first time to our knowledge, that ferroptotic death can be initiated by a common Gram-negative bacterial pathogen, $P$. aerugino$s a$, that expresses and uses pLoxA to damage bronchial epithelial cells. The detection of pLoxA in $P$. aeruginosa in the early 2000 s created an apparent paradox: this organism does not contain PUFA lipids, the substrates of pLoxA-catalyzed reactions. Overall, with the exception of several representatives of marine bacteria, mostly from Shewanella species (48), prokaryotic bacteria do not synthesize and usually do not contain PUFA lipids $(8,48)$. This in turn explains the lack of bacterial PUFA-metabolizing enzymes and respective genes in their genomes (8). Intriguingly, some bacteria, including $P$. aeruginosa, contain several genes/enzymes of PUFA metabolism $(34,49)$. These include enzymes of PUFA oxygenation and their subsequent strictly regulated conversion into different biologically active metabolites (34).

From phylogenetic studies of bacterial lipoxygenases, it can be assumed that $P$. aeruginosa was naturally selected to acquire lipoxygenase (pLoxA) because it successfully assists in the organism's metabolic diversity (adapted to grow in partial or total lack of oxygen) (50) and ubiquitous presence (soil, water, all humanmade environments, plants, animals, and humans) $(51,52)$. This interpretation is compatible with the demonstrated enhanced expression and secretion of pLoxA during biofilm growth of the pathogen (13). This facilitates the pLoxA's theft-ferroptotic mechanism as a virulence factor rather than membrane-stabilizing enzyme $(7,8)$. Transition to biofilm growth enables traits of multicellular lifestyle and "group behavior," which allow $P$. aeruginosa to thrive in a hyperinflammatory $\mathrm{CF}$ environment, resist antibiotic treatments, and evade both arms of host immune response (40, 53). The switch from planktonic to biofilm growth is accompanied by multiple but critical changes, such as development of a mucoid phenotype (54), auxotrophy (55), adapted metabolism, reduction of acute virulence factors and expression of new ones $(39,56)$, high mutation rate $(57)$, and antibiotic resistance $(53,58,59)$.

While pLoxA retains the major organizational principles essential for non-heme Fe catalysis of dioxygenation of PUFA lipids present in many mammalian and human $15 \mathrm{LOXes}$, it also acquired several specific features unique for $P$. aeruginosa's role as a bacterial pathogen $(11,12)$.pLoxA lacks the $\beta$-barrel, which is typical of mammalian 15LOXes. The $\beta$-barrel is substituted in pLoxA by PEBP1 $\alpha$-helices forming a lid, which presumably participates in substrate binding to the catalytic site via regulatory open and closed states of the protein. This function of the lid helices may be akin to the proposed role of a separate regulatory protein, PEBP1 (3), in human 15LOXs. This helical hairpin region exhibits low sequence identity among bacterial LOXes, suggesting that the observed pathogenic (or pro-ferroptotic) behavior may be specific to $P$. aeruginosa. Consistent with this, our LC-MS analysis of clinical samples revealed elevated levels of pro-ferroptotic 15-HOO-AA-PE products in CF patients infected with $P$. aeruginosa but not with other pathogens having sequences similar to pLoxA in their genomes.

The ability of $P$. aeruginosa pLoxA to act as a generator of signals for programmed death to host lung epithelial cells is in line with our previous data on the capacity of exogenous mammalian
15LOX2 + PEBP1 to trigger ferroptosis in GPX4-deficient target cells (3). Combined, these results provide evidence for the importance of 15LOX-driven PE oxidation as a pro-ferroptotic mechanism. We demonstrate that pLoxA can selectively and specifically oxidize membrane PLs, particularly AA-PE, of host epithelial cells and produce 15-HOO-AA-PE acting as pro-ferroptotic signals that, in the absence of GPX4 activity, cause ferroptosis. We demonstrate that pLoxA can selectively and specifically oxidize membrane PLs, particularly AA-PE, of host epithelial cells and produce $15-\mathrm{HOO}-$ AA-PE acting as pro-ferroptotic signals. We also show that expression of pLoxA and pro-ferroptotic activity are associated with the pro-biofilm growth conditions of $P$. aeruginosa, the major pathogen in CF. It is plausible to envisage the secretion of pLoxA in vesicles as a pathogenic strategy adopted by $P$. aeruginosa to deliver pLoxA into host cells (15). Once inside, it initiates lipid oxidation and generation of pro-ferroptotic PL hydroperoxide signals. It is also possible that accessible AA-PE of ferroptotically dying cells may be utilized by secreted pLoxA as the substrate for the production of the pro-ferroptotic signals. Therefore, once formed, the signals can propagate from cell to cell in a non-cell-autonomous fashion.

In the lung of CF patients, once $P$. aeruginosa biofilm-based chronic infection is established, it is virtually impossible to eradicate, and the infection is associated with increased mortality and morbidity (60). Similarly, in P. aeruginosa nosocomial infections, a current worldwide health care issue (42), the bacteria can grow as biofilms on abiotic and biotic surfaces, resist antibiotic treatments, and favor emergence of multidrug-resistant strains. Here we show that the role of pLoxA in the pathogenesis of $P$. aeruginosa during more persistent biofilm growth is critical. pLoxA-driven oxidation of accessible AA-PE (inside or outside injured target cells) yielding 15-HOO-AA-PEs as cell death signals propagates in a non-cell-autonomous fashion, spreading among the neighboring cells and allowing for enhanced lung colonization. It is possible that (i) this phenomenon of $P$. aeruginos $a$-induced synchronized death of host epithelial cells may produce essential building blocks for the biofilm formation, hence enhanced colonization by the pathogen; and (ii) clusters of dying epithelial cells may impair its barrier functions, thus causing increased vulnerability of host tissues to other injurious factors (e.g., in CF, sepsis). Collectively, the clinical data from CF patients and $P$. aeruginosa ICU respiratory isolates strongly suggest ferroptosis as a vital feature of $P$. aeruginosa pathogenesis and that pLoxA is indispensable for this process. Based on these findings, we propose that the development of new specific small molecule pLoxA inhibitors may lead to novel therapies against $\mathrm{CF}$ and persistent lower respiratory tract infections. This is particularly important as we demonstrated the inefficiency of selective mammalian 15LOX inhibitors in protecting against $P$. aeruginosa-induced ferroptosis.

\section{Methods}

Reagents. MEM (Thermo Fisher Scientific, 11095-080), FCS (Gibco, 10437-028), Lysogeny broth (LB) (Sigma-Aldrich, L3152), z-VADfmk (Millipore, 627610), necrostatin-1s (BioVision, 2263), bafilomycin-A1 (Sigma-Aldrich, B1793), RSL3 (Selleck Chemicals, S8155), ferrostatin-1 (Sigma-Aldrich, SML0583), PD146176 and ML351 (Cayman Chemical, 4079-26-9 and 847163-28-4), 1-stearoyl-2-arachidonoyl-sn-glycero-3-phosphoethanolamine (SAPE) (Avanti Polar Lipids, 
850804), BSA (Sigma-Aldrich), Pierce 660nm Protein Assay (Thermo Fisher Scientific, 22660), Pierce BCA Protein Assay Kit (Thermo Fisher Scientific, 23225), MTT (Thermo Fisher Scientific, M6494), arabinose (Sigma-Aldrich, A3256), deoxycholic acid (Thermo Fisher Scientific, BP349), anti-GPX4 (rabbit monoclonal, Abcam, ab125066), anti-ACSL4 (F-4) (mouse monoclonal, sc-365230) and anti-LPCAT3 (goat polyclonal, sc-161831, Santa Cruz Biotechnology Inc.), anti-actin (mouse monoclonal, Sigma-Aldrich, A3854, clone AC-15), anti-Pseudomonas aeruginosa PA-I galactophilic lectin ployclonal antibody (rabbit ployclonal, MyBioSource, MBS1495605); secondary antibodies, rabbit anti-mouse (A9044), goat anti-rabbit (A0545), and rabbit antigoat (A5420), were from Sigma-Aldrich.

Cell culture. HBE cells (a human bronchial epithelial cell line originally established by Dieter Gruenert) (61) were cultured in MEM (Thermo Fisher Scientific) supplemented with 10\% FCS (Gibco), 50 $\mathrm{U} / \mathrm{ml}$ penicillin-streptomycin (Thermo Fisher Scientific), plasmocin (InvivoGen), and L-glutamine (Thermo Fisher Scientific). HBE cells until passage 50 were used for experiments.

Bacterial strains. P. aeruginosa WT (MPAO1), loxA::Tn (PA1169 transposon deletion mutant) (PW3111), and hyper-biofilm mutant $(\triangle w s p F)$ strains were obtained from the P. aeruginosa transposon mutant library, University of Washington (Seattle, Washington, USA) (62). PAO1 isogenic strain, rugose small colony variant MJK8, and its

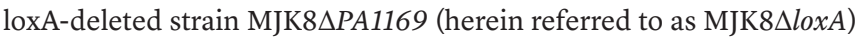
were generated as previously described (16).

$P$. aeruginosa ICU respiratory isolates. Tobramycin-resistant P. aeruginosa respiratory isolates from ICUs were identified at the clinical microbiology laboratory of University of Pittsburgh Medical Center (IRB no. PRO12060302) and designated TRPA002-TRPA122. For confirmation, the isolates were reidentified as $P$. aeruginosa using a commercial biochemical panel (API 20NE system, bioMérieux).

loxA::Tn complementation. PW3111 (MPAO1 loxA::Tn) was complemented with an arabinose-inducible promoter at the chromosomal attTn7 site as previously described using pUC18-miniTn7T-Gm-GW (63). From genomic MPAO1 DNA, PA119 was amplified using primers attB5-RBS-loxA-F and attB2-loxAdown-R (Supplemental Table 4), which included the entire lox $A$ coding region and introduced a synthetic ribosomal binding site. The loxA amplicon was gel purified and recombined into pDONR221P5P2 (Supplemental Table 4) using BP Clonase II (Invitrogen). The resulting entry vector, pENTR221L5L2::RBS-PA1169, was transformed into E. coli DH5 $\alpha$, and insertions were checked by PCR and Sanger sequencing (Eurofins) using primers M13F(-21) and M13R. We used MultiSite Gateway Technology (Invitrogen) to recombine pENTR221L1L5r::araC- $\mathrm{P}_{\mathrm{BAD}}$ (the plasmid pENTR221L1L5r::araC- $\mathrm{P}_{\mathrm{BAD}}$ was a gift from Joe Harrison, Department of Biological Sciences, University of Calgary, Calgary, Alberta, Canada) and pENTR221L5L2::RBS-PA1169 with pUC18miniTn7T-Gm-GW, generating the complementation vector pUC18miniTn7T:: $\mathrm{P}_{\mathrm{BAD}}-P A 1169$. The complementation vector was transformed into E. coli $\mathrm{DH} 5 \alpha$, and plasmids from transformants were sequenced using primers pBAD-F and pUC18BackboneR01. To complement PW3111, we electroporated pUC18miniTn7T:: $\mathrm{P}_{\mathrm{BAD}}-\mathrm{PA1169}$ and pTNS3 into PW3111. Transformants were selected on LB with 30 $\mu \mathrm{g} / \mathrm{ml}$ gentamicin, and colony PCR was performed using primers $\mathrm{P}_{\text {Tn7R }}$ and $\mathrm{P}_{\text {glmsdown }}$ as previously described (63) to identify an insertion at the attTn7 site on the chromosome (Supplemental Table 4). All the E. coli strains, plasmids, and primers used for complementation studies are shown in Supplemental Table 4.
Abiotic biofilm and supernatant collection. P. aeruginosa strains including the tobramycin-resistant clinical isolates were grown overnight in $\mathrm{LB}$ at $37^{\circ} \mathrm{C}, 220 \mathrm{rpm}$. These overnight cultures were diluted in MEM medium (without phenol red) to an $\mathrm{OD}_{600}$ of 0.05 and plated in 96-well vinyl microtiter plates (100 $\mu$ l per well) (Costar). Bacteria were grown for 24 hours at $37^{\circ} \mathrm{C}$ without agitation, and the supernatant was collected by centrifugation at 3,000 $g$ for 8 minutes and then frozen before further use in cell death and activity assays. For pLoxA activity assays and treatment of HBE cells, frozen supernatants were thawed and centrifuged at 3,000 $g$ for 8 minutes, and protein concentration was estimated by the BCA protein assay (Thermo Fisher Scientific). Supernatants were added to HBE cells containing fresh MEM medium and incubated for 20 hours followed by cell death assays. We believe this freeze-thaw step was sufficient to prevent survival of any bacteria in the supernatants (Supplemental Figure 11). In all experiments, control was untreated HBE cells containing media only. Overnight cultures of MJK8 and MJK84loxA were resuspended vigorously before measuring the $\mathrm{OD}_{600}$. CFU assay was performed to determine the number of bacteria $/ \mathrm{ml}$ in the supernatants of the P. aeruginosa WT, $\triangle w s p F$, and lox A::Tn strains. For this, the supernatants were serially diluted in MEM medium (without phenol red), plated on LB agar plates, and incubated overnight at $37^{\circ} \mathrm{C}$. For biofilm quantification, after the removal of supernatant, biofilms were washed using deionized water to remove nonadherent bacteria and stained with crystal violet (41\%) for 15 minutes at room temperature (RT). Samples were washed (3 times), and biofilms were resuspended in 30\% acetic acid and quantified at $\mathrm{OD}_{550}$ as previously reported (64).

For pLoxA imaging, biofilms were grown on glass coverslips by placing the coverslip vertically in 12 -well plates containing WT, $\triangle w s p F$, or loxA::Tn biofilm cultures. After incubation $\left(37^{\circ} \mathrm{C}\right.$ for 24 hours), the coverslips were washed with water (2 times), fixed with paraformaldehyde (2\%) or glutaraldehyde (2.5\%) for 30 minutes, and then used for pLoxA staining or for SEM imaging.

Biotic biofilm assay. HBEs were enzymatically dissociated, expanded in growth media, seeded onto Transwell inserts, and grown at the air-liquid interface for 7-10 days. Cultures were used when well polarized and differentiated (65). Biotic biofilm assays were performed as follows: Overnight culture of the $\Delta w s p F$ mutant strain $P$. aeruginosa was rinsed once and added to the apical surface of the epithelial cells in $500 \mu \mathrm{l}$ MEM with $0.4 \%$ glutamine at an MOI of 25 . After the 60-minute bacterial attachment period, developing biofilms were treated with vehicle or ferrostatin-1 $(0.2$ and $1.0 \mu \mathrm{M})$ for the subsequent 5 hours. At the end of this period, biofilms were removed with $0.1 \%$ Triton $\mathrm{X}-100$, and live bacteria were plated in a CFU assay.

KD of ACSL4 and LPCAT3. HBE cells were transfected with a mix of 2 Dicer substrate siRNAs (DsiRNAs) against ACSL4 (mm. Ri.Acsl4.13. and mm.Ri.Acsl4.13.2) or ON-TARGET plus SMART pool LPCAT3 siRNA (L-010273-01-0005, Dharmacon) or with negative control DsiRNA (51-01-14-04, Integrated DNA Technologies) using Lipofectamine 3000 (Life Technologies) according to the manufacturer's instructions. Efficiency of KD was determined by Western blotting using antibodies against ACSL4 (1:1,000) and LPCAT3 (1:500).

Live cell imaging. To visualize intracellular lipid peroxidation, HBE cells were seeded in glass-bottom (35- $\mathrm{mm})$ tissue culture dishes (MatTek Corp.), and after 48 hours, cells were prestained with Liperfluo (Dojindo Molecular Technologies Inc., $10 \mu \mathrm{M}$ ) for 30 minutes, washed, and then treated with supernatant collected under biofilm 
growth conditions from the $\triangle w s p F$ strain or with RSL3 (200 nM). The dish was inserted into a closed, thermo-controlled $\left(37^{\circ} \mathrm{C}\right)$ stage top incubator (Tokai Hit Co.) atop the motorized stage of an inverted Nikon TiE fluorescent microscope equipped with a $60 \times$ oil immersion optic (Nikon, CFI Plan Fluor, NA 1.43) and NIS Elements Software. Liperfluo was excited using a diode-pumped light engine (SPECTRA $\mathrm{X}$, Lumencor) and detected using an ORCA-Flash 4.0 sCMOS camera (Hamamatsu) and excitation and emission filters from Chroma. Data were collected every 5 minutes for 4 hours, on approximately 10-20 cells per stage position, with 10-15 stage positions in each of 4 separate experiments per condition. Data were analyzed using NIS Elements (Nikon). Fluorescence of the region of the image without cells was used for background subtraction.

To monitor cell death, HBE cells were seeded in glass-bottom partitioned $(35 \mathrm{~mm})$ cell culture dishes (Greiner Bio-One), and after 48 hours, cells were treated with $\triangle w s p F$ supernatant in the presence of SYTOX green and examined for 48 hours by live cell imaging. Data were collected every 30 minutes for 48 hours and analyzed by NIS Elements.

Western blotting. Total cell lysates were prepared after washing cells with PBS and then adding cell lysis buffer ( $25 \mathrm{mM}$ Tris- $\mathrm{HCl}, \mathrm{pH}$ 7.5, $150 \mathrm{mM} \mathrm{NaCl}$, and 1\% SDS) or RIPA buffer (Thermo Fisher Scientific) containing freshly added protease-phosphatase cocktail inhibitor mix (Thermo Fisher Scientific). Samples were boiled at $95^{\circ} \mathrm{C}$ for 10 minutes and sonicated to break down DNA. Total protein was estimated by BCA protein assay kit (Pierce, 23225) and diluted with $2 \times$ or $4 \times$ Laemmli buffer before loading in Tris-glycine gradient gels (8\%-16\%, Life Technologies). Proteins were transferred to a PVDF membrane (Bio-Rad), blocked with milk (5\%) in PBST (0.1\% Tween) for 1 hour, incubated with primary antibodies (1\% BSA in PBST) overnight at RT, washed 3 times, and incubated with secondary antibody (1 hour) in blocking solution before developing with SuperSignal West Pico Chemiluminescent Substrate (Thermo Fisher Scientific).

For analysis of GPX4, HBE cells (80,000 cells per well) were seeded in 6-well plates, and samples were collected after 20 hours of treatment. Quantification of the bands was performed using NIH ImageJ software.

For pLoxA Western blotting, $P$. aeruginosa (WT, $\triangle w s p F$, loxA::Tn), MJK8 (WT and MJK84loxA), and the clinical isolates were grown in biofilm conditions in $3 \mathrm{ml}$ MEM media or LB media for 24 hours. After centrifugation, pellet was washed with PBS and then resuspended in RIPA lysis buffer or in Laemmli buffer $(2 \times)$. Cells were lysed by freezethaw (3 cycles) and sonication (3 times, 10 seconds on, 10 seconds off). Supernatants were collected after centrifugation (10 minutes, $13,400 \mathrm{~g}$ ), and the amount of protein was estimated as mentioned above. Samples in Laemmli buffer were boiled (10 minutes) and sonicated, and protein was estimated using Pierce 660nm Protein Assay. Equal amount of protein was loaded in Tris-glycine gradient gels and transferred to PVDF or nitrocellulose membranes (Bio-Rad), blocked with BSA (2.5\%) in PBST 2 hours, and incubated with anti-pLoxA antibody $(1: 1,000)$ (in 1\% BSA in PBST) overnight at RT. Blots were washed (3 times) and incubated for 1 hour with secondary antibody $(1: 2,000)$ in milk (5\%) before developing. Bands were quantified by Image J software, and recombinant pLoxA included in the gels was used to generate a standard curve and determine the amount of pLoxA in clinical isolates.

AA supplementation. AA (Sigma-Aldrich, A0281) was added to HBE cells as reported previously (1). Briefly, suspension of AA was incubated with fatty acid-free BSA (5\%) to form a complex of AA with BSA. This soluble complex was added to and incubated with HBE cells overnight along with WT, $\triangle w s p F$, or loxA::Tn supernatants containing $10 \mu \mathrm{g}$ protein, before estimating the cell death by flow cytometry.

Cell death assay. PI (Life Technology) staining was used to measure cell death using flow cytometry as described previously (1). Briefly, HBE cells were plated in 24-well plates (12,000-15,000 cells/well). After 48 hours, cells in fresh media were incubated with $P$. aeruginosa supernatants containing $10 \mu \mathrm{g}$ protein alone or with the indicated treatments for 20 hours. Cells were trypsinized, centrifuged at $700 \mathrm{~g}$ for 6 minutes, resuspended in PBS containing PI for 5 minutes on ice, and then monitored by flow cytometry. The gate settings were chosen at $5 \%$ or less PI-positive cells for control samples. Annexin V-FITC (BioVision) staining and MTT (Invitrogen) cell viability assay were used as described previously (1). Cell death was calculated as per the manufacturer's instructions.

For ACSL4- and LPCAT3-KD samples, 12 hours after transfection, cells were seeded in 24-well plates (15,000 per well) and in a 6 -cm tissue culture dish. After 36 hours, cells were treated with $\Delta w s p F$ supernatant for 20 hours, and cell death was analyzed by flow cytometry after staining with PI. Samples from the 6-cm dish were used for Western blotting.

Cell death induced by genetically manipulated pLoxA. Overnight culture of pLoxA-deficient (loxA::Tn [PW3111]) and complemented (PW3111 Tn7::loxA) strains were reinoculated at an $\mathrm{OD}_{600}$ of 0.05 and grown in biofilm conditions in the presence of $0.1 \%$ arabinose for 24 hours. Cultures were centrifuged, and the pellet was washed with PBS, then resuspended in PBS containing protease inhibitors. Cells were lysed by the freeze-thaw method as mentioned above. Cell supernatants were collected after centrifugation (10 minutes, 13,400 g), and the protein amount was estimated by BCA protein assay (Thermo Fisher Scientific, 23225). An equal amount of protein $(100 \mu \mathrm{g})$ was incubated with SAPE $(100 \mu \mathrm{M})$ in $150 \mathrm{mM}$ borate buffer (pH 9.0) containing $0.02 \%$ deoxycholate for 30 minutes at RT under a constant supply of oxygen. The reaction mix was added to RSL3-pretreated (20 $\mathrm{nM}$ ) HBE cells (4 hours) with or without ferrostatin-1 and incubated for 2 hours in the absence of serum. Then serum was added back to the final concentration of $10 \%$, and the cells were incubated for 20 hours before cell death measurements.

Ferroptosis induced by exogenous 15-HOO-AA-PE. HBE cells were pretreated with RSL3 (20 nM) for 4 hours and then incubated with 15-HOO-AA-PE $(0.75 \mu \mathrm{M})$ for 2 hours in the absence of serum. After adding back serum (10\% final concentration), 15-HOO-AA-PE-sensitized cells were then treated with equal amounts of supernatant $(5 \mu \mathrm{g})$ from either the loxA::Tn or WT strain in the presence or absence of ferrostatin-1 and incubated overnight before cell death assays.

GSH measurements. After incubations, cells were suspended in 80 $\mu \mathrm{PBS}$, freeze-thawed at $-80^{\circ} \mathrm{C}$, and mildly sonicated on ice. For GSH analysis (run in triplicate) $15 \mu \mathrm{l}$ sample was incubated in PBS with 10 $\mathrm{mM}$ Thiol Fluorescent Probe IV (Millipore) for 15 minutes. Then fluorescence was measured with a Cytation 5 imaging reader (BioTek) by using an excitation wavelength of $400 \mathrm{~nm}$ and an emission wavelength of $465 \mathrm{~nm}$. Protein concentration was measured by Bradford Protein Assay (Bio-Rad).

Assessment of pyocyanin. UV-visible spectroscopy of chloroform extracts from clinical isolates was performed in the range of 300-800 $\mathrm{nm}$. The total concentration of pyocyanin was determined in chloroform extracts dried and re-dissolved in methanol by absorbance at $690 \mathrm{~nm}$ using a molar extinction coefficient of 5,816 (66). 
$A A$ and $A A-P E$ docking to $p L o X A$. Ligand docking was performed using the SMINA package (67), which is customized to better support scoring function and high-performance energy minimization. The structure used for pLoxA was 4G32 (12). Five runs each for AA and AA-PE were performed, and the top binding pose (lowest energy) was considered for statistical purposes.

Sequence alignment, generation of sequence similarity map, and phylogenetic tree construction. A multiple sequence alignment (MSA) of lipoxygenase domains was downloaded from the Pfam protein families database (33) and analyzed using ProDy (68). The MSA was refined by removal of poorly aligned and highly similar sequences, resulting in a final alignment of 218 sequences that was more amenable to further analysis. This was followed by alignment and sorting of the sequences with MUSCLE $(69,70)$ and manual curation using UniPro UGENE (71). The resulting alignment was then fed back into ProDy for additional analysis, including production of the sequence identity matrix shown in Figure 4A.

GNM and anisotropic network model analyses for protein dynamics. Anisotropic network model (ANM) (72) and GNM (73) analysis was done using the ProDY API. Identification of relative modes of motion along the principal mode was done using GNM. In this model, the positive (red) and negative (blue) regions indicate the structural regions that are subject to correlated (positive) or anticorrelated (negative) motions. The theory of GNM and ANM can be found in ref. 71 and ref. 72 , respectively. The movie was generated using the ANM implemented in the DynOmics server (74). Structural visualization and analysis were performed using ProDy.

Expression and purification of pLoxA. pLoxA with a $\mathrm{His}_{6}$ tag was purified using NTA-Ni affinity chromatography, as described previously (3, 13). Briefly, pet151/His6-pLoxA plasmid containing E. coli BL21 (DE3) was grown to an $\mathrm{OD}_{600}$ of 0.6 at $37^{\circ} \mathrm{C}$ and induced by incubating the culture at low temperature $\left(20^{\circ} \mathrm{C}\right)$ overnight. Pellet was collected by centrifugation $(5,000 \mathrm{~g}, 10$ minutes $)$, resuspended in buffer A ( $25 \mathrm{mM}$ HEPES [pH 7.5], $150 \mathrm{mM} \mathrm{NaCl}$ ), and sonicated to lyse the cells. Lysate was centrifuged at 40,000 $g$ for 25 minutes, and supernatant was loaded onto an NTA-Ni affinity column and washed with lysis buffer, and the protein was eluted with a gradient of 0 to $500 \mathrm{mM}$ imidazole in buffer $\mathrm{A}$. Fractions containing pLoxA were pooled together and preserved in $10 \%$ (v/v) glycerol and stored at $-80^{\circ} \mathrm{C}$ for further use.

pLoxA antibody purification. pLoxA antibody against recombinant purified pLoxA was raised and purified from rabbit whole blood by Pocono Rabbit Farm and Laboratory as described previously (13). Briefly, recombinant pLoxA ( $3 \mathrm{ml}$ of $2 \mathrm{mg} / \mathrm{ml}$ ) was immobilized using Pierce AminoLink immobilization kit following the manufacturer's recommendations. After loading $4 \mathrm{ml}$ rabbit serum, column was washed with PBS (7 ml), $1 \mathrm{M} \mathrm{NaCl}(1 \mathrm{ml})$, and PBS (7 ml) and finally eluted with glycine $(6 \mathrm{ml})$. Fractions with more than $0.05 \mathrm{mg} / \mathrm{ml}$ of protein were pooled and stored at $-80^{\circ} \mathrm{C}$ in the presence of $10 \%$ glycerol.

Liposome preparation. Liposomes of 1,2-dioleoyl-PC (DOPC)/ SAPE (1:1) or DOPC/AA (1:10) were prepared using a sonication method. Briefly, DOPC and SAPE (Avanti Polar Lipids Inc.) lipids were dried under nitrogen and resuspended in PBS ( $\mathrm{pH}$ 7.4) prepared fresh under oxygen flow. Lipids were vortexed and then sonicated using Torbeo ultra cell disruptor (36180 series; Cole Parmer) with maximum power for 10 minutes on ice.

Oxidation of $A A$ and $A A-P E$ by $P$. aeruginosa supernatant or pLoxA in a model system. Lipoxygenase activity of the $P$. aeruginosa supernatants (WT, $\triangle w s p F$, and loxA::Tn) was assessed by formation of primary products of AA oxidation - 15-HpETE or PE (C18:0/15HpETE) - that were detected by reverse-phase LC-MS. Briefly, supernatants were incubated with liposomes (DOPC/AA or DOPC/ SAPE) in the presence of $0.5 \mu \mathrm{M} 15-\mathrm{HpETE}, 100 \mu \mathrm{M}$ DTPA in 20 mM HEPES pH 7.4 at $37^{\circ} \mathrm{C}$ for 10 minutes. To prevent conversion of 15-HpETE to secondary products during incubation, HEPES buffer included $100 \mu \mathrm{M}$ DTPA (for transition metal chelation) that had been saturated with oxygen (before addition of supernatants or pLoxA). For oxidation of AA-PE with purified recombinant pLoxA, $400 \mathrm{nM}$ enzyme was incubated with DOPC/SAPE (1:1) liposomes along with $0.5 \mu \mathrm{M} 15$-HpETE, $100 \mu \mathrm{M}$ DTPA in $20 \mathrm{mM}$ HEPES $\mathrm{pH}$ 7.4 at $37^{\circ} \mathrm{C}$ for 10 minutes. At the end of incubation, AA and PE as well as their oxygenated products were extracted by the Folch (75) procedure and analyzed by LC-MS.

Determination of pLoxA activity in P. aeruginosa clinical isolates. Clinical isolates $(50 \mu \mathrm{l})$ were mixed in a 1:1 ratio with $20 \mathrm{mM}$ HEPES buffer, pH 7.4, containing $100 \mu \mathrm{M}$ DTPA, $10 \mu \mathrm{M}$ AA (arachidonic acid), and $0.5 \mu \mathrm{M} 15-\mathrm{HOO}-\mathrm{AA}-\mathrm{PE}$ and incubated at $37^{\circ} \mathrm{C}$ during 10 minutes. At the end of incubation, AA and its oxygenated products were extracted by Folch procedure (75) and analyzed by LC-MS. The data were normalized to the amount of protein and presented as $\mathrm{AA}_{\text {oxidation }}$ ( $\mathrm{pmol} / \mathrm{min} / \mu \mathrm{g}$ of protein).

Redox phospholipidomics. MS of PLs was performed on an Orbitrap Fusion Lumos mass spectrometer (Thermo Fisher Scientific). PLs were separated on a normal phase column (Luna $3 \mu \mathrm{m}$ Silica [2] $100 \AA$, $150 \times 2.0 \mathrm{~mm}$; Phenomenex) at a flow rate of $0.2 \mathrm{ml} / \mathrm{min}$ on a Dionex UltiMate 3000 HPLC system at $35^{\circ} \mathrm{C}$. Gradient solvents A (propanol/hexane/water, 285:215:5, v/v/v) and B (propanol/hexane/water, 285:215:40, v/v/v) containing $10 \mathrm{mM}$ ammonium acetate were used. The column was eluted for 0-23 minutes with a linear gradient from $10 \%$ to $32 \% \mathrm{~B} ; 23-32$ minutes from $32 \%$ to $65 \% \mathrm{~B} ; 32-35$ minutes from $65 \%$ to $100 \% \mathrm{~B} ; 35-62$ minutes held at $100 \% \mathrm{~B} ; 62-64$ minutes from $100 \%$ to $10 \%$ B, followed by and equilibration from $64-80$ minutes at $10 \% \mathrm{~B}$. The instrument was operated with ESI probe in negative mode. Analysis of data was performed using the software package Compound Discoverer (Thermo Fisher Scientific) with an in-house-generated analysis workflow and oxidized PL database. Lipids were filtered by retention time and confirmed by fragmentation analysis.

Analysis of $A A$ and its oxidation products. AA and AAox were analyzed by LC-MS using Dionex UltiMate TM 3000 HPLC system coupled on-line to a Q-Exactive Hybrid Quadrupole-Orbitrap mass spectrometer (Thermo Fisher Scientific) using a C18 column (Acclaim PepMap RSLC, $0.3 \mathrm{~mm} \times 15 \mathrm{~cm}$, Thermo Fisher Scientific). Gradient solvents A (20\% methanol/80\% water, v/v) and B (90\% methanol/10\% water, v/v) with $5 \mathrm{mM}$ ammonium acetate were used. The column was eluted at a flow rate of $12 \mu \mathrm{l} / \mathrm{min}$ using a linear gradient from $30 \%$ to $95 \%$ solvent B over 70 minutes, held at $95 \%$ B from 70 to 80 minutes, followed by a return to initial conditions by 83 minutes and re-equilibration for an additional 7 minutes. Spectra were acquired in negative mode with the scan range of $150-600 \mathrm{~m} / \mathrm{z}$, maximum injection time $100 \mathrm{~ms}$, and resolution of 1,400,000. An isolation window of 1.0 Da was set for the MS and MS2 scans with an inclusion list of AA oxygenated products. Capillary spray voltage was set at $2.6 \mathrm{kV}$, and capillary temperature was $250^{\circ} \mathrm{C}$. The S-lens RF level was set to 60 . Analytical data were acquired and analyzed using Xcalibur software. 
Statistics. All data are shown as mean \pm SD from at least 3 experiments (biological replicates) unless otherwise specified. Statistical analysis was performed using SPSS software 25 (IBM). One-way ANOVA followed by Fisher's least significant difference post hoc analysis was used for multiple-group comparisons. The threshold of significance was set at $P<0.05$. Sample sizes $(n)$ are indicated in the figure legends. In the box plots, the boundaries of the box represent the first quartile (bottom boundary) and third quartile (top boundary); the band represents the median; and the whiskers represent the maximum and minimum values.

For correlation analysis, first we checked whether a Box-Cox transformation (76) was necessary to make the percent of ferroptotic cell death variable normally distributed, using PROC TRANSREG in SAS 9.4 software. It was found that the data could be regarded as normally distributed after being raised to the power of 0.25 . We studied the effect of the following factors on the percent of ferroptotic cell death: pLoxA amount, pLoxA activities (15LOX activity and 5LOX activity), amount of pyocyanin in $P$. aeruginosa supernatants, and GSH content in HBE cells after treatments. First, the univariate effect of each factor was studied with the linear regression for the transformed percent of ferroptotic cell death. Variables from univariate models that had a $P$ value of less than 0.1 were considered for inclusion in the multivariate regression analysis. Then we performed the multivariate analysis, using the stepwise variable selection method to find a best linear regression model for this transformed end point. In the stepwise procedure, variables were removed from the model if the associated $P$ value was greater than 0.05 . All $P$ values reported are 2-sided.

Study approval. Samples were obtained from individuals who provided written informed consent prior to their participation in the study. All studies were approved by the IRB of the University of Pittsburgh. ICU respiratory isolates were obtained at the clinical microbiology laboratory through IRB no. PRO12060302.

\section{Author contributions}

HHD, JMB, HB, and VEK conceived the study. HHD, EB, HCT, GM, and BAF performed experiments with cells. HHD analyzed the data. YYT and HCT performed MS measurements and analyzed data. VAT performed pLoxA model experiments and LC-MS analysis. AK performed GSH and pyocyanin measurements. JSL and YD selected clinical isolates and participated in experiments with their testing. KMR and IS performed computational modeling. JK performed phylogenetic and evolutionary analysis. IB supervised computational and phylogenetic studies. CMSC and SW performed imaging experiments and participated in interpreting them. AFO, BAF, and JRG prepared $P$. aeruginosa supernatants. CRA and JMB generated complementation strain. CJF and TRH purified recombinant pLoxA and pLoxA antibody. HW analyzed data and performed correlation analysis. TSA discussed and interpreted MS results. MRP provided MJK8 and MJK84loxA strains and interpreted the results. JMP acquired samples from $\mathrm{CF}$ patients and discussed and interpreted the results. HB, JSG, RKM, and TRH participated in formulating the idea and interpreting the data. YYT, IB, JSL, and HB participated in writing the manuscript. HHD and VEK wrote the manuscript.

\section{Acknowledgments}

This work was supported by the NIH (U19AI068021, HL11445306, NS076511, NS061817, HL136143, HL086884, HL123771, and DK072506) and by Russian academic excellence project “5-100.”

Address correspondence to: Hülya Bayır, Department of Environmental and Occupational Health, Public Health Building, 130 De Soto St., Pittsburgh, Pennsylvania 15261, USA. Phone: 412.692.5164; Email: bayihx@ccm.upmc.edu. Or to: Valerian E. Kagan, Department of Environmental and Occupational Health, Public Health Building, 130 De Soto St., Pittsburgh, Pennsylvania 15261, USA. Phone: 412.624.9700; Email: kagan@pitt.edu.
1. Kagan VE, et al. Oxidized arachidonic and adrenic PEs navigate cells to ferroptosis. Nat Chem Biol. 2017;13(1):81-90.

2. Yang WS, Kim KJ, Gaschler MM, Patel M, Shchepinov MS, Stockwell BR. Peroxidation of polyunsaturated fatty acids by lipoxygenases drives ferroptosis. Proc Natl Acad Sci U S A. 2016;113(34):E4966-E4975.

3. Wenzel SE, et al. PEBP1 Wardens ferroptosis by enabling lipoxygenase generation of lipid death signals. Cell. 2017;171(3):628-641.e26.

4. Kuhn H, Walther M, Kuban RJ. Mammalian arachidonate 15-lipoxygenases structure, function, and biological implications. Prostaglandins Other Lipid Mediat. 2002;68-69:263-290.

5. Oliw EH. Plant and fungal lipoxygenases. Prostaglandins Other Lipid Mediat. 2002;68-69:313-323.

6. Haeggström JZ, Funk CD. Lipoxygenase and leukotriene pathways: biochemistry, biology, and roles in disease. Chem Rev. 2011;111(10):5866-5898.

7. Hansen J, Garreta A, Benincasa M, Fusté MC, Busquets M, Manresa A. Bacterial lipoxygenases, a new subfamily of enzymes? A phylogenetic approach. Appl Microbiol Biotechnol.
2013;97(11):4737-4747.

8. Horn T, et al. Evolutionary aspects of lipoxygenases and genetic diversity of human leukotriene signaling. Prog Lipid Res. 2015;57:13-39.

9. Vance RE, Hong S, Gronert K, Serhan CN, Mekalanos JJ. The opportunistic pathogen Pseudomonas aeruginosa carries a secretable arachidonate 15-lipoxygenase. Proc Natl Acad Sci U S A. 2004;101(7):2135-2139.

10. Banthiya S, Pekárová M, Kuhn H, Heydeck D. Secreted lipoxygenase from Pseudomonas aeruginosa exhibits biomembrane oxygenase activity and induces hemolysis in human red blood cells. Arch Biochem Biophys. 2015;584:116-124.

11. Banthiya S, et al. Structural and functional basis of phospholipid oxygenase activity of bacterial lipoxygenase from Pseudomonas aeruginosa. Biochim Biophys Acta. 2016;1861(11):1681-1692.

12. Garreta A, et al. Structure and interaction with phospholipids of a prokaryotic lipoxygenase from Pseudomonas aeruginosa. FASEB J. 2013;27(12):4811-4821.

13. Deschamps JD, et al. Biochemical and cellular characterization and inhibitor discovery of pseudomonas aeruginosa 15-lipoxygenase. Biochemis- try. 2016;55(23):3329-3340.

14. Ballok AE, Filkins LM, Bomberger JM, Stanton BA, O'Toole GA. Epoxide-mediated differential packaging of Cif and other virulence factors into outer membrane vesicles. J Bacteriol. 2014;196(20):3633-3642.

15. Bomberger JM, Maceachran DP, Coutermarsh BA, Ye S, O'Toole GA, Stanton BA. Long-distance delivery of bacterial virulence factors by Pseudomonas aeruginosa outer membrane vesicles. PLoS Pathog. 2009;5(4):e1000382.

16. Starkey M, et al. Pseudomonas aeruginosa rugose small-colony variants have adaptations that likely promote persistence in the cystic fibrosis lung. J Bacteriol. 2009;191(11):3492-3503.

17. Smith EE, et al. Genetic adaptation by Pseudomonas aeruginosa to the airways of cystic fibrosis patients. Proc Natl Acad Sci US A. 2006;103(22):8487-8492.

18. Traverse CC, Mayo-Smith LM, Poltak SR, Cooper VS. Tangled bank of experimentally evolved Burkholderia biofilms reflects selection during chronic infections. Proc Natl Acad Sci U S A. 2013;110(3):E250-E259.

19. Ou Y, Wang SJ, Li D, Chu B, Gu W. Activation of SAT1 engages polyamine metabolism with 
p53-mediated ferroptotic responses. Proc Natl Acad Sci U S A. 2016;113(44):E6806-E6812.

20. Ousingsawat J, et al. Ca2+ signals, cell membrane disintegration, and activation of TMEM16F during necroptosis. Cell Mol Life Sci. 2017;74(1):173-181.

21. Redmann M, et al. Inhibition of autophagy with bafilomycin and chloroquine decreases mitochondrial quality and bioenergetic function in primary neurons. Redox Biol. 2017;11:73-81.

22. Rai G, et al. Discovery of ML351, a potent and selective inhibitor of human 15-lipoxygenase-1. In Probe Reports from the NIH Molecular Libraries Program (2010). Bethesda, Maryland, USA: National Center for Biotechnology Information (US); 2010-. https://www.ncbi.nlm.nih.gov/ pubmed/24672829. Updated January 13, 2014. Accessed September 5, 2018.

23. Yamanaka K, et al. A novel fluorescent probe with high sensitivity and selective detection of lipid hydroperoxides in cells. RSC Adv. 2012;2:7894-7900.

24. Cozza G, et al. Glutathione peroxidase 4-catalyzed reduction of lipid hydroperoxides in membranes: the polar head of membrane phospholipids binds the enzyme and addresses the fatty acid hydroperoxide group toward the redox center. Free Radic Biol Med. 2017;112:1-11.

25. Linkermann A, et al. Synchronized renal tubular cell death involves ferroptosis. Proc Natl Acad Sci U S A. 2014;111(47):16836-16841.

26. Kim SE, et al. Ultrasmall nanoparticles induce ferroptosis in nutrient-deprived cancer cells and suppress tumour growth. Nat Nanotechnol. 2016;11(11):977-985.

27. Poursaitidis I, et al. Oncogene-Selective sensitivity to synchronous cell death following modulation of the amino acid nutrient cystine. Cell Rep. 2017;18(11):2547-2556.

28. Küch EM, et al. Differentially localized acylCoA synthetase 4 isoenzymes mediate the metabolic channeling of fatty acids towards phosphatidylinositol. Biochim Biophys Acta. 2014;1841(2):227-239.

29. Li LO, Klett EL, Coleman RA. Acyl-CoA synthesis, lipid metabolism and lipotoxicity. Biochim Biophys Acta. 2010;1801(3):246-251.

30. Thomas JP, Maiorino M, Ursini F, Girotti AW. Protective action of phospholipid hydroperoxide glutathione peroxidase against membranedamaging lipid peroxidation. In situ reduction of phospholipid and cholesterol hydroperoxides. J Biol Chem. 1990;265(1):454-461.

31. Yang WS, et al. Regulation of ferroptotic cancer cell death by GPX4. Cell. 2014;156(1-2):317-331.

32. Gaschler MM, et al. FINO2 initiates ferroptosis through GPX4 inactivation and iron oxidation. Nat Chem Biol. 2018;14(5):507-515.

33. Finn RD, et al. The Pfam protein families database: towards a more sustainable future. Nucleic Acids Res. 2016;44(D1):D279-D285.

34. Flitter BA, et al. Pseudomonas aeruginosa sabotages the generation of host proresolving lipid mediators. Proc Natl Acad Sci U S A. 2017;114(1):136-141.

35. Marvig RL, Sommer LM, Molin S, Johansen HK. Convergent evolution and adaptation of Pseu- domonas aeruginosa within patients with cystic fibrosis. Nat Genet. 2015;47(1):57-64.

36. Folkesson A, et al. Adaptation of Pseudomonas aeruginosa to the cystic fibrosis airway: an evolutionary perspective. Nat Rev Microbiol. 2012;10(12):841-851.

37. Gillmor SA, Villaseñor A, Fletterick R, Sigal $\mathrm{E}$, Browner MF. The structure of mammalian 15-lipoxygenase reveals similarity to the lipases and the determinants of substrate specificity. Nat Struct Biol. 1997;4(12):1003-1009.

38. Kobe MJ, Neau DB, Mitchell CE, Bartlett SG, Newcomer ME. The structure of human 15-lipoxygenase-2 with a substrate mimic. J Biol Chem. 2014;289(12):8562-8569.

39. Bragonzi A, et al. Pseudomonas aeruginosa microevolution during cystic fibrosis lung infection establishes clones with adapted virulence. Am J Respir Crit Care Med. 2009;180(2):138-145.

40. Moradali MF, Ghods S, Rehm BH. Pseudomonas aeruginosa lifestyle: a paradigm for adaptation, survival, and persistence. Front Cell Infect Microbiol. 2017;7:39.

41. Vincent JL, et al. Sepsis in European intensive care units: results of the SOAP study. Crit Care Med. 2006;34(2):344-353.

42. Vincent JL, et al. International study of the prevalence and outcomes of infection in intensive care units. JAMA. 2009;302(21):2323-2329.

43. Global priority list of antibiotic-resistant bacteria to guide research, discovery and development of new antibiotics. World Health Organization. http://www.who.int/medicines/publications/ global-priority-list-antibiotic-resistant-bacteria/ en/. Published February 27, 2017. Accessed August 10, 2018.

44. Hofmans S, et al. Novel ferroptosis inhibitors with improved potency and ADME properties. JMed Chem. 2016;59(5):2041-2053.

45. Friedmann Angeli JP, et al. Inactivation of the ferroptosis regulator Gpx4 triggers acute renal failure in mice. Nat Cell Biol. 2014;16(12):1180-1191.

46. Hambright WS, Fonseca RS, Chen L, Na R, Ran Q. Ablation of ferroptosis regulator glutathione peroxidase 4 in forebrain neurons promotes cognitive impairment and neurodegeneration. Redox Biol. 2017;12:8-17.

47. Distéfano AM, et al. Heat stress induces ferroptosis-like cell death in plants. J Cell Biol. 2017;216(2):463-476.

48. Russell NJ, Nichols DS. Polyunsaturated fatty acids in marine bacteria - a dogma rewritten. Microbiology (Reading, Engl).1999;145( Pt 4):767-779.

49. Aldrovandi M, et al. Specific oxygenation of plasma membrane phospholipids by Pseudomonas aeruginosa lipoxygenase induces structural and functional alterations in mammalian cells. Biochim Biophys Acta. 2018;1863(2):152-164.

50. Silby MW, Winstanley C, Godfrey SA, Levy SB, Jackson RW. Pseudomonas genomes: diverse and adaptable. FEMS Microbiol Rev. 2011;35(4):652-680.

51. Frimmersdorf E, Horatzek S, Pelnikevich A, Wiehlmann L, Schomburg D. How Pseudomonas aeruginosa adapts to various environments: a metabolomic approach. Environ Microbiol. 2010;12(6):1734-1747
52. Lyczak JB, Cannon CL, Pier GB. Establishment of Pseudomonas aeruginosa infection: lessons from a versatile opportunist. Microbes Infect. 2000;2(9):1051-1060.

53. Hogardt M, Heesemann J. Microevolution of Pseudomonas aeruginosa to a chronic pathogen of the cystic fibrosis lung. Curr Top Microbiol Immunol. 2013;358:91-118.

54. Govan JR, Deretic V. Microbial pathogenesis in cystic fibrosis: mucoid Pseudomonas aeruginosa and Burkholderia cepacia. Microbiol Rev. 1996;60(3):539-574.

55. Barth AL, Pitt TL. The high amino-acid content of sputum from cystic fibrosis patients promotes growth of auxotrophic Pseudomonas aeruginosa. JMed Microbiol. 1996;45(2):110-119.

56. Nguyen D, Singh PK. Evolving stealth: genetic adaptation of Pseudomonas aeruginosa during cystic fibrosis infections. Proc Natl Acad Sci U S A. 2006;103(22):8305-8306.

57. Oliver A, Cantón R, Campo P, Baquero F, Blázquez J. High frequency of hypermutable Pseudomonas aeruginosa in cystic fibrosis lung infection. Science. 2000;288(5469):1251-1254.

58. Hogardt M, Heesemann J. Adaptation of Pseudomonas aeruginosa during persistence in the cystic fibrosis lung. Int J Med Microbiol. 2010;300(8):557-562.

59. Breidenstein EB, de la Fuente-Núñez C, Hancock RE. Pseudomonas aeruginosa: all roads lead to resistance. Trends Microbiol. 2011;19(8):419-426.

60. Langton Hewer SC, Smyth AR. Antibiotic strategies for eradicating Pseudomonas aeruginosa in people with cystic fibrosis. Cochrane Database Syst Rev. 2009;(4):CD004197.

61. Gruenert DC, Willems M, Cassiman JJ, Frizzell RA. Established cell lines used in cystic fibrosis research. JCyst Fibros. 2004;3(Suppl 2):191-196.

62. Jacobs MA, et al. Comprehensive transposon mutant library of Pseudomonas aeruginosa. Proc Natl Acad Sci U S A. 2003;100(24):14339-14344.

63. Choi KH, Schweizer HP. mini-Tn7 insertion in bacteria with single attTn7 sites: example Pseudomonas aeruginosa. Nat Protoc. 2006;1(1):153-161.

64. O'Toole GA, Kolter R. Initiation of biofilm formation in Pseudomonas fluorescens WCS365 proceeds via multiple, convergent signalling pathways: a genetic analysis. Mol Microbiol. 1998;28(3):449-461.

65. Zemke AC, et al. Nitrite modulates bacterial antibiotic susceptibility and biofilm formation in association with airway epithelial cells. Free Radic Biol Med. 2014;77:307-316.

66. Muller M, Merrett ND. Mechanism for glutathione-mediated protection against the Pseudomonas aeruginosa redox toxin, pyocyanin. Chem Biol Interact. 2015;232:30-37.

67. Koes DR, Baumgartner MP, Camacho CJ. Lessons learned in empirical scoring with smina from the CSAR 2011 benchmarking exercise. JChem Inf Model. 2013;53(8):1893-1904.

68. Bakan A, et al. Evol and ProDy for bridging protein sequence evolution and structural dynamics. Bioinformatics. 2014;30(18):2681-2683.

69. Edgar RC. MUSCLE: a multiple sequence align- 
ment method with reduced time and space complexity. BMC Bioinformatics. 2004;5:113.

70. Edgar RC. MUSCLE: multiple sequence alignment with high accuracy and high throughput. Nucleic Acids Res. 2004;32(5):1792-1797.

71. Okonechnikov K, Golosova O, Fursov M, UGENE team. Unipro UGENE: a unified bioinformatics toolkit. Bioinformatics. 2012;28(8):1166-1167.
72. Eyal E, Lum G, Bahar I. The anisotropic network model web server at 2015 (ANM 2.0). Bioinformatics. 2015;31(9):1487-1489.

73. Li H, Chang YY, Yang LW, Bahar I. iGNM 2.0: the Gaussian network model database for biomolecular structural dynamics. Nucleic Acids Res. 2016;44(D1):D415-D422.

74. Li H, Chang YY, Lee JY, Bahar I, Yang LW. DynOm- ics: dynamics of structural proteome and beyond. Nucleic Acids Res. 2017;45(W1):W374-W380.

75. Folch J, Lees M, Sloane Stanley GH. A simple method for the isolation and purification of total lipides from animal tissues. J Biol Chem. 1957;226(1):497-509.

76. Box GEP, Cox DR. An analysis of transformations. JRoyal Stat Soc Series B. 1964;26(2):211-252. 